Article

\title{
Double Parametric Fuzzy Numbers Approximate Scheme for Solving One-Dimensional Fuzzy Heat-Like and Wave-Like Equations
}

\author{
Ali Fareed Jameel ${ }^{1, *(\mathbb{D})}$, Sarmad A. Jameel Altaie ${ }^{1,2}$, Sardar Gul Amen Aljabbari ${ }^{1,3}$, \\ Abbas AlZubaidi ${ }^{4}\left(\mathbb{D}\right.$ and Noraziah Haji Man ${ }^{1}$ \\ 1 School of Quantitative Sciences, College of Arts and Sciences, Universiti Utara Malaysia (UUM), Sintok, \\ Kedah 06010, Malaysia; sarmad_a@ahsgs.uum.edu.my or 120024@uotechnology.edu.iq (S.A.J.A.); \\ sardar_gul_amen@ahsgs.uum.edu.my (S.G.A.A.); noraziah@uum.edu.my (N.H.M.) \\ 2 Computer Engineering Department, University of Technology, Baghdad 10066, Iraq \\ 3 Department of Financial and Banking, College of Business Administration and Financial Science, \\ Al-Kitab University, Altun Kupri, Kirkuk 36015, Iraq \\ 4 Biomedical Engineering Division, University of Saskatchewan, Saskatoon, SK S7N 5C9, Canada; \\ aba658@usask.ca \\ * Correspondence: alifareed@uum.edu.my
}

Received: 16 September 2020; Accepted: 5 October 2020; Published: 10 October 2020

\begin{abstract}
This article discusses an approximate scheme for solving one-dimensional heat-like and wave-like equations in fuzzy environment based on the homotopy perturbation method (HPM). The concept of topology in homotopy is used to create a convergent series solution of the fuzzy equations. The objective of the study is to formulate the double parametric fuzzy HPM to obtain approximate solutions of fuzzy heat-like and fuzzy wave-like equations. The fuzzification and the defuzzification analysis for the double parametric form of fuzzy numbers of the fuzzy heat-like and the fuzzy wave-like equations is carried out. The proof of convergence of the solution under the developed approximate scheme is provided. The effectiveness of the proposed method is tested by numerically solving examples of fuzzy heat-like and wave-like equations where results indicate that the approach is efficient not only in terms of accuracy but also with respect to CPU time consumption.
\end{abstract}

Keywords: fuzzy partial differential equation; fuzzy heat-like equation; fuzzy wave-like equation; homotopy perturbation method (HPM); fuzzy numbers

\section{Introduction}

The principles of fuzzy sets theory have been illustrated and applied in many fields [1,2]. Over the last years, the use of fuzzy sets in computational mathematics has gained particular attention, in order to properly revise existing techniques for solving differential equations from a fuzzy theory standpoint e.g., [3,4]. In particular, fuzzy partial differential equations (FPDEs) are known to be useful in modeling a dynamic system with inadequate knowledge about the behavior of the system, where they incorporate uncertainty characteristics into the model [5,6]. Due to their frequent role in the design and simulation of many technological applications, such as heat transmission and mass transfer, electromagnetic fields, static and dynamic structure, meteorology, and biomechanics, FPDEs have gained great interest among scientists and engineers [7-9].

Some uncertainty models involving FPDES that have been studied include fuzzy heat-like equations and fuzzy wave-like equations in [10-17]. Several scholars have addressed the approximate-analytical methods of finding solutions of different types FPDEs under the single parametric form of fuzzy number properties [17-20]. Homotopy Perturbation Method (HPM) is 
an approximate-analytical method that has been used by many scientists and engineers to determine the solution of linear and nonlinear models approximately [21-25]. Solving equations by HPM provides an elegantly computable solution in a fast-convergent series, without the need for discretization and linearity that are required in numerical schemes [25].

However, with the existing single parametric fuzzy approach of approximate-analytical methods, it is known that the analysis and computational work is complex, where an $\mathrm{n} x \mathrm{n}$ completely fuzzy system has to be transformed into a much larger $2 \mathrm{n} \times 2 \mathrm{n}$ crisp system. To alleviate this complexity issue, the double parametric approach, which is not only more general and simple but also requires less analysis and computational work, has been developed in studies on FPDEs involving beam equations and fuzzy delay differential equations [26,27]. The incorporation of additional embedding parameter that functions as a deforming parameter annihilates the necessity to transform the $n \times n$ completely fuzzy system to a larger system in the solving process but instead enables practitioners to maintain the same $\mathrm{n} \times \mathrm{n}$ crisp system order after transformation, which, in turn, reduces the entire work complexity.

Therefore, the aims of the paper is to develop an approximate-analytical scheme based on the double parametric fuzzy HPM to obtain approximate solutions of one-dimensional fuzzy heat-like and fuzzy wave-like equations in the form of double-parametric fuzzy numbers and also to prove the convergence of the obtained solutions. We wish to point out that the emphasis in this paper is on the computational and formal issues; a discussion on the physical meaning of the fuzzy solutions to partial differential equations can be retrieved in [28].

In this paper, definitions of the single parametric form and the double parametric form of fuzzy numbers are recapitulated in Section 2. Sections 3 and 4, describe the defuzzification analysis under the double parametric form of fuzzy numbers for the fuzzy heat-like and the fuzzy wave-like equations respectively. In Section 5, a general form of HPM in the double parametric form of fuzzy numbers for solving fuzzy differential equations is presented. Sections 6 and 7 , the focus is to reformulate HPM in the double parametric form of fuzzy numbers for solving fuzzy heat-like and the fuzzy wave-like equations respectively. The theorem of convergence is provided in Section 8. Section 9 gives the findings and discussion of test examples in solving fuzzy heat-like and fuzzy wave-like equations, and this is followed by the conclusion of the study. Note that some of the basic fuzzy definitions, remarks, and concepts that are not described in this paper are standard and well-known. The notion of fuzzy number and its arithmetic operations, fuzzy functions, fuzzy level sets, and fuzzy Zadeh expansion theory are described in [29-33].

\section{Fuzzy Numbers}

2.1. Triangular Fuzzy Numbers [34]: A triangular fuzzy number is a fuzzy number $\tilde{R}$ which is defined by the numbers $\alpha, \beta$ and $\gamma(\alpha<\beta<\gamma)$ and its graph is a triangle with the vertex at $x=\beta$ and the base on the $[\alpha, \beta]$ interval as shown in Figure 1. For simplicity, the left threshold value $\alpha$, the midpoint $\beta$ and the right threshold value $\gamma$ are used to represent a triangular fuzzy number $\tilde{R}(\alpha, \beta, \gamma)$ where its membership function of $\tilde{R}$ is given as follows:

$$
\mu(x, \alpha, \beta, \gamma)=\left\{\begin{array}{c}
0, \text { if } x<\alpha \\
\frac{x-\alpha}{\beta-\alpha}, \text { if } \alpha \leq x \leq \beta \\
\frac{\gamma-x}{\gamma-\beta}, \text { if } \beta \leq x \leq y \\
0, \text { if } x>\gamma
\end{array}\right.
$$

where $\mu(x, \alpha, \beta, \gamma)$ is called the membership function and its $r$-level sets are denoted by:

$$
[\mu]_{r}=[\alpha+r(\beta-\alpha), \gamma-r(\gamma-\beta)], \text { for } r \in[0,1] .
$$




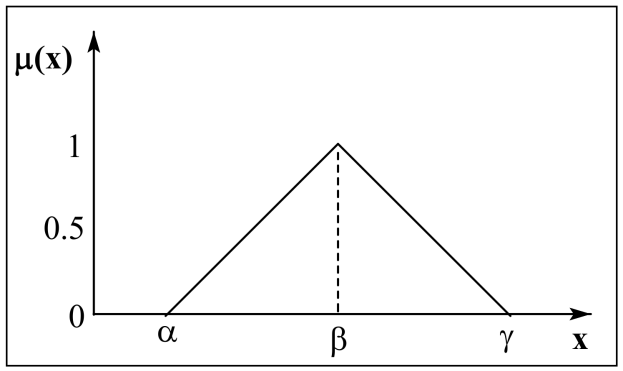

Figure 1. Triangular Fuzzy Number.

In addition, the $r$-level (or $r$-cut) sets of a fuzzy set $\tilde{R}$, labeled as $\tilde{R}_{r}$, is the crisp set of all $x \in T$ such that for the membership function $\tilde{\mu}_{r} \geq r$, we have $\tilde{\mu}_{r}=\left\{x \in T \mid \tilde{\mu}_{r}>r, r \in[0,1]\right\}$ [34].

The r-level set is the relation between the fuzzy domain and the crisp domain. We are able to use the benefits of crisp domain and fuzzy domain theories to the effect that

$$
\frac{x-\alpha}{\beta-\alpha}=r \rightarrow r(\beta-\alpha)=x-\alpha \rightarrow x=\alpha+r(\beta-\alpha),
$$

the lower bound of fuzzy number, and

$$
\frac{\gamma-x}{\gamma-\beta}=r \rightarrow r(\gamma-\beta)=\gamma-x \rightarrow x=\gamma-r(\gamma-\beta),
$$

the upper bound of fuzzy number. For the purpose of this paper, we only provide the definition of double parametric form of fuzzy numbers and its properties, while for the single parametric form, the reader can refer to [26] for further details.

2.2. Double parametric form of fuzzy numbers [26]: Let $\tilde{\mu}=[\underline{\mu}(r), \bar{\mu}(r)]$ be a parametric form of fuzzy number $\tilde{R}$; then one may represent the double parametric form in crisp values as $\tilde{\mu}(r, b)=b[\bar{\mu}(r)-\underline{\mu}(r)]+\underline{\mu}(r)$, where $r, b \in[0,1]$. The embedding parameter $b$ denotes the deforming parameter such that if $b=0$ then $\tilde{\mu}(r, 0)=\mu(r)$ (lower bound fuzzy number), and if $b=1$ then $\tilde{\mu}(r, 1)=\bar{\mu}(r)$ (upper bound fuzzy number). In this way, the computational time of the double parametric form will be less than the computational time of the single parametric form.

\section{Fuzzy Heat-Like Equation Analysis}

In this section, we present the defuzzification analysis of fuzzy heat-like equation with variable coefficients under H-partial differentiability [30] by using the definition of double parametric form of fuzzy number by consider the one-dimensional fuzzy heat-like equations with fuzzy initial condition [10].

$$
\begin{aligned}
& \tilde{u}_{t}(x, t)+\tilde{\theta}_{1} \tilde{g}(x) \tilde{u}_{x x}(x, t)=\tilde{\theta}_{2} \tilde{s}(x, t), \\
& \tilde{u}(x, 0)=\tilde{\theta}_{3} \tilde{c}(x), 0<x<l, 0<t<T .
\end{aligned}
$$

where $\tilde{u}(x, t), \tilde{g}(x)$ and $\tilde{s}(x, t)$ are defined as fuzzy functions of the crisp variables $x$ and $t$ for all $r, b[0,1]$ such that:

$$
\begin{gathered}
\tilde{u}(x, t, r, b)=[\tilde{u}(x, t, r)]_{b}=b[\bar{u}(x, t, r)-\underline{u}(x, t, r)]+\underline{u}(x, t, r), \\
\tilde{g}(x, r, b)=[\tilde{g}(x, r)]_{b}=b[\bar{g}(x, r)-\underline{g}(x, r)]+\underline{g}(x, r), \\
\tilde{s}(x, t, r, b)=[\tilde{s}(x, t, r)]_{b}=b[\bar{s}(x, t, r)-\underline{s}(x, t, r)]+\underline{s}(x, t, r) . \\
{\left[\tilde{\theta}_{1}(r)\right]_{b}=b\left[\bar{\theta}_{1}(r)-\underline{\theta}_{1}(r)\right]+\underline{\theta}_{1}(r),} \\
{\left[\tilde{\theta}_{2}(r)\right]_{b}=b\left[\bar{\theta}_{2}(r)-\underline{\theta}_{2}(r)\right]+\underline{\theta}_{2}(r), \text { and }}
\end{gathered}
$$




$$
\left[\tilde{\theta}_{3}(r)\right]_{b}=b\left[\bar{\theta}_{3}(r)-\underline{\theta}_{3}(r)\right]+\underline{\theta}_{3}(r) .
$$

The fuzzy H-partial derivatives of Equation (1) defuzzification are presented as follows:

$$
\begin{gathered}
{\left[\tilde{u}_{t}(x, t, r)\right]_{b}=b\left[\bar{u}_{t}(x, t, r)-\underline{u}_{t}(x, t, r)\right]+\underline{u}_{t}(x, t, r),} \\
{\left[\tilde{u}_{x x}(x, t, r)\right]_{b}=b\left[\bar{u}_{x x}(x, t, r)-\underline{u}_{x x}(x, t, r)\right]+\underline{u}_{x x}(x, t, r) .}
\end{gathered}
$$

Also the defuzzification of the fuzzy initial condition is given by:

$$
[\tilde{u}(x, 0, r)]_{b}=b[\bar{u}(x, t, r)-\underline{u}(x, t, r)]+\underline{u}(x, t, r) .
$$

Applying the concept of Zadeh extension principle [30], to obtain the following relations:

$$
\begin{aligned}
& \underline{u}(x, t, r)=\min \{\tilde{u}(t, x, \tilde{\mu}(r)) \mid \tilde{\mu}(r) \in \tilde{u}(x, t, r)\}, \\
& \bar{u}(x, t, r)=\max \{\tilde{u}(t, x, \tilde{\mu}(r)) \mid \tilde{\mu}(r) \in \tilde{u}(x, t, r)\},
\end{aligned}
$$

where $\tilde{\mu}(r)=[\underline{\mu}(r), \bar{\mu}(r)]$ is the membership function of the triangular fuzzy number such that

$$
\tilde{u}(x, t, \tilde{\mu}(r))=[\underline{u}(x, t, \underline{\mu}(r), \bar{\mu}(r)), \bar{u}(x, t, \underline{\mu}(r), \bar{\mu}(r))] .
$$

According definition 2.2, we define the double parametric form of Equation (1) is defined in the following relation for all $r, b \in[0,1]$ :

$$
\begin{aligned}
b\left[\bar{u}_{t}(x, t, r)-\underline{u}_{t}(x, t, r)\right]+\underline{u}_{t}(x, t, r) & \\
& =b\left[\bar{\theta}_{1}(r) \bar{g}(x, r) \bar{u}_{x x}(x, t, r)-\underline{\theta}_{1}(r) \underline{g}(x, r) \underline{u}_{x x}(x, t, r)\right] \\
& +\underline{\theta}_{1}(r) g(x, r) \underline{u}_{x x}(x, t, r) \\
& =b\left[\bar{\theta}_{2}(r) \bar{s}(x, t, r)-\underline{\theta}_{2}(r) \underline{s}(x, t, r)\right]+\underline{\theta}_{2}(r) \underline{s}(x, t, r)
\end{aligned}
$$

subject to the initial condition

$$
b[\bar{u}(x, 0, r)-\underline{u}(x, 0, r)]+\underline{u}(x, 0, r)=b\left[\bar{\theta}_{3}(r) \bar{c}(x, r)-\underline{\theta}_{3}(r) \underline{c}(x, r)\right]+\underline{\theta}_{3}(r) \underline{c}(x, r) .
$$

\section{Fuzzy Wave-Like Equation Analysis}

Also in this section, we follow the defuzzification analysis steps of Section 3 for the analysis of fuzzy wave-like with variable coefficients under H-partial differentiability by using the definition of the double parametric form of fuzzy numbers by consider the one-dimensional fuzzy wave-like equation with fuzzy initial conditions [14]:

$$
\begin{gathered}
\tilde{u}_{t t}(x, t)+\tilde{\eta}_{1} \tilde{h}(x) \tilde{u}_{x x}(x, t)=\tilde{\eta}_{2} \tilde{w}(x, t), \\
\tilde{u}(x, 0)=\tilde{\eta}_{3} \tilde{d}(x), \tilde{u}_{t}(0, x)=\tilde{\eta}_{4} \tilde{q}(x) 0<x<l, 0<t<T,
\end{gathered}
$$

where $\tilde{u}(t, x), \tilde{h}(x)$, and $\tilde{w}(t, x)$ are defined as fuzzy functions of the crisp variables $x$ and $t$ for all $r, b[0,1]$ such that:

$$
\begin{gathered}
{[\tilde{u}(x, t, r)]_{b}=b[\bar{u}(x, t, r)-\underline{u}(x, t, r)]+\underline{u}(x, t, r),} \\
{[\tilde{h}(x, r)]_{\beta}=b[\bar{h}(x, r)-\underline{h}(x, r)]+\underline{h}(x, r),} \\
{[\tilde{w}(x, t, r)]_{b}=b[\bar{w}(x, t, r)-\underline{w}(x, t, r)]+\underline{w}(x, t, r) .} \\
{\left[\tilde{\eta}_{1}(r)\right]_{b}=b\left[\bar{\eta}_{1}(r)-\underline{\eta}_{1}(r)\right]+\underline{\eta}_{1}(r),}
\end{gathered}
$$




$$
\begin{gathered}
{\left[\tilde{\eta}_{2}(r)\right]_{b}=b\left[\bar{\eta}_{2}(r)-\underline{\eta}_{2}(r)\right]+\underline{\eta}_{2}(r), \text { and }} \\
{\left[\tilde{\eta}_{3}(r)\right]_{b}=b\left[\bar{\eta}_{3}(r)-\underline{\eta}_{3}(r)\right]+\underline{\eta}_{3}(r) .}
\end{gathered}
$$

The fuzzy H-partial derivatives of Equation (3) defuzzification are presented as follows:

$$
\begin{aligned}
{\left[\tilde{u}_{t i}(x, t, r)\right]_{b} } & =b\left[\bar{u}_{t i}(x, t, r)-\underline{u}_{t i}(x, t, r)\right]+\underline{u}_{t i}(x, t, r), \\
{\left[\tilde{u}_{x x}(x, t, r)\right]_{b} } & =b\left[\bar{u}_{x x}(x, t, r)-\underline{u}_{x x}(x, t, r)\right]+\underline{u}_{x x}(x, t, r) .
\end{aligned}
$$

Also the defuzzification of the fuzzy initial conditions is given by:

$$
\begin{gathered}
{[\tilde{u}(x, 0, r)]_{b}=b[\bar{u}(x, 0, r)-\underline{u}(x, 0, r)]+\underline{u}(x, 0, r), \text { and }} \\
{\left[\tilde{u}_{t}(x, 0, r)\right]_{b}=b\left[\bar{u}_{t}(x, t, r)-\underline{u}_{t}(x, t, r)\right]+\underline{u}_{t}(x, t, r) .}
\end{gathered}
$$

From the defuzzification analysis in Section 3, the double parametric form of Equation (3) is defined in the following relation for all $r, b \in[0,1]$ :

$$
\begin{aligned}
b\left[\bar{u}_{t t}(x, t, r)-\underline{u}_{t t}(x, t, r)\right]+\underline{u}_{t t}(x, t, r) & \\
& =b\left[\bar{\eta}_{1}(r) \bar{h}(x, r) \bar{u}_{x x}(x, t, r)-\underline{\eta}_{1}(r) \underline{h}(x, r) \underline{u}_{x x}(x, t, r)\right] \\
& +\underline{\eta}_{1}(r) \underline{h}(x, r) \underline{u}_{x x}(x, t, r) \\
& =b\left[\bar{\eta}_{2}(r) \bar{w}(x, t, r)-\underline{\eta}_{2}(r) \underline{w}(x, t, r)\right]+\underline{\eta}_{2}(r) \underline{w}(x, t, r)
\end{aligned}
$$

subject to the initial conditions

$$
\begin{gathered}
b[\bar{u}(x, 0, r)-\underline{u}(x, 0, r)]+\underline{u}(x, 0, r)=b\left[\bar{\eta}_{3}(r) \bar{d}(x, r)-\underline{\eta}_{3}(r) \underline{d}(x, r)\right]+\underline{\eta}_{3}(r) \underline{d}(x, r), \\
{\left[\tilde{u}_{t}(x, 0, r)\right]_{b}=b\left[\bar{u}_{t}(x, t, r)-\underline{u}_{t}(x, t, r)\right]+\underline{u}_{t}(x, t, r)=b\left[\bar{\eta}_{4}(r) \bar{q}(x, r)-\underline{\eta}_{4}(r) \underline{q}(x, r)\right]+\underline{\eta}_{4}(r) \underline{q}(x, r) .}
\end{gathered}
$$

\section{General Double Parametric Formula of Fuzzy HPM}

From [23], the general HPM formula for solving a crisp differential equation is extended to the fuzzy double parametric form of HPM. Based on [18] and the definition in 2.2, we consider the general fuzzy differential equation in the double parametric form as follows:

$$
\left\{\begin{array}{c}
A[\tilde{u}]_{r}^{b}+[\tilde{f}(s)]_{r}^{b}=0, s \in \Omega . \\
\mathcal{B}\left(\tilde{u}, \frac{\partial \tilde{u}}{\partial n}\right)=0, s \in \Gamma .
\end{array}\right.
$$

where

$$
\begin{aligned}
{[\tilde{u}]_{r}^{b} } & =b[\bar{u}(r)-\underline{u}(r)]+\underline{u}(r), \text { and } \\
{[f]_{r}^{b} } & =b[\bar{f}(s, r)-\underline{f}(s, r)]+\underline{f}(s, r) .
\end{aligned}
$$

In Equation (5), the boundary operator is denoted by $\mathcal{B}$, the domain $\Omega$ includes the boundary $\Gamma$, $A$ is a general differential operator, and $[\tilde{f}(t)]_{r}^{b}$ is the known fuzzy analytical function. According to [23], operator $A$ can be separated into two components, $L$ and $N$, where $L$ is a linear operator and $N$ is a nonlinear operator. Consequently, Equation (5) can be rewritten as follows:

$$
L[\tilde{u}]_{r}^{b}+N[\tilde{u}]_{r}^{b}+[\tilde{f}(s)]_{r}^{b}=0 .
$$


We create a homotopy form using the homotopy technique as mentioned in $[5,7,12,13]$ such that for each $b, r \in[0,1]$ the relation $[\tilde{v}(s ; p)]_{r}^{b}: \Omega \times[0,1] \rightarrow \mathbb{R}$ satisfies the following:

$$
\mathcal{H}(\tilde{v}(s, r, b), p)=\left[L[\tilde{v}(s)]_{r}^{b}-L\left[\tilde{u}_{0}(s)\right]_{r}^{b}+p L\left[\tilde{u}_{0}(s)\right]_{r}^{b}\right]+p\left[N\left([\tilde{v}(s)]_{r}^{b}\right)+[\tilde{f}(s)]_{r}^{b}\right]=0 .
$$

where

$$
\begin{gathered}
{\left[\tilde{u}_{0}(s)\right]_{r}^{b}=b\left[\bar{u}_{0}(s, r)-\underline{u}_{0}(s, r)\right]+\underline{u}_{0}(s, r), \text { and }} \\
{[\tilde{v}(s)]_{r}^{b}=b[\bar{v}(s, r)-\underline{v}(s, r)]+\underline{v}(s, r) .}
\end{gathered}
$$

The initial guess starting function of Equation (1) denoted by $\left[\tilde{u}_{0}(s)\right]_{r}^{b}$ satisfies the equation boundary conditions, and the parameter $p \in[0,1]$ is an embedding parameter to generate double parametric form of HPM series solution [24]. Now, Equation (6) can be rewritten as follows:

$$
\left\{\begin{array}{c}
\mathcal{H}(\tilde{v}(s, r, b), 0)=L[\tilde{v}(s)]_{r}^{b}-L\left[\tilde{u}_{0}(s)\right]_{r}^{b}=0 \\
\mathcal{H}(\tilde{v}(s, r, b), 1)=A[\tilde{v}(s)]_{r}^{b}-[\tilde{f}(s)]_{r}^{b}=0 .
\end{array}\right.
$$

According to HPM in [23], the solution of Equation (5) is represented by power series form:

$$
\tilde{v}(s, r, b)=\sum_{i=0}^{\infty} p^{i} \tilde{v}_{i}(s, r, b)=\sum_{i=0}^{\infty} p^{i}\left(b\left[\bar{v}_{i}(s, r)-\underline{v}_{i}(s, r)\right]+\underline{v}_{i}(s, r)\right)
$$

where

$$
\begin{gathered}
\tilde{v}_{i}(s, r, b)=\tilde{v}_{0}(s, r, b)+p \tilde{v}_{1}(s, r, b)+p^{2} \tilde{v}_{2}(s, r, b)+\ldots, \\
b[\bar{v}(s, r)-\underline{v}(s, r)]+\underline{v}(s, r)=b\left[\bar{v}_{0}(s, r)-\underline{v}_{0}(s, r)\right]+\underline{v}_{0}(s, r)+ \\
p\left(b\left[\bar{v}_{1}(s, r)-\underline{v}_{1}(s, r)\right]+\underline{v}_{1}(s, r)\right)+p^{2}\left(b\left[\bar{v}_{2}(s, r)-\underline{v}_{2}(s, r)\right]+\underline{v}_{2}(s, r)\right)+\ldots
\end{gathered}
$$

Through Equation (10), we can obtain the multi fuzzy series solutions by setting the values of double parameter $b$ from 0 to 1 , since, if we set $b=0$, we obtain the lower fuzzy solution of Equation (5) as

$$
\underline{v}(s, r)=\underline{v}_{0}(s, r)+p \underline{v}_{1}(s, r)+p^{2} \underline{v}_{2}(s, r)+\ldots
$$

If $b=1$, we obtain the upper fuzzy solution of Equation (3) as

$$
\bar{v}(s, r)=\bar{v}_{0}(s, r)+\bar{v}_{1}(s, r)+p^{2} \bar{v}_{2}(s, r)+\ldots
$$

The approximate solution of Equation (3) is obtained by setting $p=1$, that is,

$$
\tilde{u}(s, r, b)=\lim _{p \rightarrow 1} \tilde{v}(s, r, b)=\tilde{v}_{0}(s, r, b)+\tilde{v}_{1}(s, r, b)+\tilde{v}_{2}(s, r, b)+\ldots
$$

\section{Double Parametric HPM Fuzzy Heat-Like Equation Analysis}

The analysis of HPM fuzzy heat-like equation in Equation (1) under as fuzzy number in double parametric form is discussed in this section. Based on analysis in Sections 3 and 4, Equation (1) can be rewritten as follows:

$$
\begin{gathered}
H(x, p, r, b)=(1-p)\left\{b\left[\left(\frac{\partial}{\partial t} \bar{u}(x, t, r)-\frac{\partial}{\partial t} \bar{u}_{0}(x, t, r)\right)-\left(\frac{\partial}{\partial t} \underline{u}(x, t, r)-\frac{\partial}{\partial t} \underline{u}_{0}(x, t, r)\right)\right]+\right. \\
\left.\left(\frac{\partial}{\partial t} \underline{u}(x, t, r)-\frac{\partial}{\partial t} \underline{u}_{0}(x, t, r)\right)\right\}+p\left[b\left(\frac{\partial}{\partial t} \bar{u}(x, t, r)-\frac{\partial}{\partial t} \underline{u}(x, t, r)\right)+\frac{\partial}{\partial t} \underline{u}(x, t, r)+\right. \\
b\left(\bar{\theta}_{1}(r) \bar{g}(x, r) \frac{\partial^{2}}{\partial x^{2}} \bar{u}(x, t, r)-\underline{\theta}_{1}(r) \underline{g}(x, r) \frac{\partial^{2}}{\partial x^{2}} \bar{u}(x, t, r)\right)+\underline{\theta}_{1}(r) \underline{g}(x, r) \frac{\partial^{2}}{\partial x^{2}} \bar{u}(x, t, r)- \\
\left.b\left(\bar{\theta}_{2}(r) \bar{s}(x, t, r)-\underline{\theta}_{2}(r) \underline{s}(x, t, r)\right)-\underline{\theta}_{2}(r) \underline{s}(x, t, r)\right]=0 .
\end{gathered}
$$


Then, the power series in $p$ representing the solution of Equation (12) can be written as

$$
\tilde{u}(x, t, r, b)=\sum_{i=0}^{\infty} p^{i} \tilde{u}_{i}(x, t, r, b) .
$$

The substitution of Equation (13) into Equation (12) yields the following:

$$
\begin{gathered}
H(x, p, r, b)=\left\{b \left[\left(\frac{\partial}{\partial t} \sum_{i=0}^{\infty} p^{i} \bar{u}_{i}(x, t, r)-\frac{\partial}{\partial t} \bar{u}_{0}(x, t, r)\right)-\left(\frac{\partial}{\partial t} \sum_{i=0}^{\infty} p^{i} \underline{u}_{i}(x, t, r)-\right.\right.\right. \\
\left.\left.\left.\frac{\partial}{\partial t} \underline{u}_{0}(x, t, r)\right)\right]+\left(\frac{\partial}{\partial t} \sum_{i=0}^{\infty} p^{i} \underline{u}_{i}(x, t, r)-\frac{\partial}{\partial t} \underline{u}_{0}(x, t, r)\right)\right\}=p\left[-b\left(\frac{\partial}{\partial t} \bar{u}_{0}(x, t, r)-\right.\right. \\
\left.\frac{\partial}{\partial t} \underline{u}_{0}(x, t, r)\right)+\frac{\partial}{\partial t} \underline{u}_{0}(x, t, r)+b\left(\bar{\theta}_{1}(r) \bar{g}(x, r) \frac{\partial^{2}}{\partial x^{2}} \sum_{i=0}^{\infty} p^{i} \bar{u}_{i}(x, t, r)-\right. \\
\left.\theta_{1}(r) g(x, r) \frac{\partial^{2}}{\partial x^{2}} \sum_{i=0}^{\infty} p^{i} \bar{u}_{i}(x, t, r)\right)+\theta_{1}(r) g(x, r) \frac{\partial^{2}}{\partial x^{2}} \sum_{i=0}^{\infty} p^{i} \underline{u}_{i}(x, t, r)- \\
\left.b\left(\bar{\theta}_{2}(r) \bar{s}(x, t, r)-\underline{\theta}_{2}(r) \underline{s}(x, t, r)\right)+\underline{\theta}_{2}(r) \underline{s}(x, t, r)\right]=0 .
\end{gathered}
$$

The initial approximation function of Equation (14) that fulfills the initial conditions of Equation (1) is specified by the following form:

$$
\tilde{u}_{0}(x, t, r, b)=b\left[\bar{u}_{0}(x, t, r)-\underline{u}_{0}(x, t, r)\right]+\underline{u}_{0}(x, t, r)=b\left[\bar{\theta}_{3}(r) \bar{c}(x, r)-\underline{\theta}_{3}(r) \underline{c}(x, r)\right]+\underline{\theta}_{3}(r) \underline{c}(x, r) .
$$

Now, both sides with similar powers of $p$ are compared to obtain the solution, and, for simplicity, we assume the initial guess is $\tilde{u}_{0}(x, t, r, b)=\tilde{u}_{0}(x, 0, r, b)$. Thus, the following recurrent relation is obtained:

$$
\begin{gathered}
p^{1}: \tilde{u}_{1}(x, t, r, b)=\int_{0}^{T}\left[-b\left(\bar{\theta}_{1}(r) \bar{g}(x, r) \frac{\partial^{2}}{\partial x^{2}} \bar{u}_{0}(x, t, r)-\underline{\theta}_{1}(r) \underline{g}(x, r) \frac{\partial^{2}}{\partial x^{2}} \underline{u}_{0}(x, t, r)\right)-\right. \\
\underline{\theta}_{1}(r) \underline{g}(x, r) \frac{\partial^{2}}{\partial x^{2}} \underline{u}_{0}(x, t, r)+b\left(\bar{\theta}_{2}(r) \bar{s}(x, t, r)-\underline{\theta}_{2}(r) \underline{s}(x, t, r)\right)+ \\
\left.\underline{\theta}_{2}(r) \underline{s}(x, t, r)\right] d t, \tilde{u}_{1}(x, 0, r, b)=0, \\
p^{2}: \tilde{u}_{2}(x, t, r, b)=\int_{0}^{T}\left[-b\left(\bar{\theta}_{1}(r) \bar{g}(x, r) \frac{\partial^{2}}{\partial x^{2}} \bar{u}_{1}(x, t, r)-\underline{\theta}_{1}(r) \underline{g}(x, r) \frac{\partial^{2}}{\partial x^{2}} \underline{u}_{1}(x, t, r)\right)\right. \\
\left.-\underline{\theta}_{1}(r) \underline{g}(x, r) \frac{\partial^{2}}{\partial x^{2}} \underline{u}_{1}(x, t, r)\right] d t, \tilde{u}_{2}(x, 0, r, b)=0, \\
p^{3}: \tilde{u}_{3}(x, t, r, b)=\int_{0}^{T}\left[-b\left(\bar{\theta}_{1}(r) \bar{g}(x, r) \frac{\partial^{2}}{\partial x^{2}} \bar{u}_{2}(x, t, r)-\underline{\theta}_{1}(r) \underline{g}(x, r) \frac{\partial^{2}}{\partial x^{2}} \underline{u}_{2}(x, t, r)\right)\right. \\
\left.-\underline{\theta}_{1}(r) \underline{g}(x, r) \frac{\partial^{2}}{\partial x^{2}} \underline{u}_{2}(x, t, r)\right] d t, \tilde{u}_{3}(x, 0, r, b)=0, \\
\ldots \\
p^{k+1}: \tilde{u}_{k}(x, t, r, b)=\int_{0}^{T}\left[-b\left(\bar{\theta}_{1}(r) \bar{g}(x, r) \frac{\partial^{2}}{\partial x^{2}} \bar{u}_{k}(x, t, r)-\underline{\theta}_{1}(r) \underline{g}(x, r) \frac{\partial^{2}}{\partial x^{2}} \underline{u}_{k}(x, t, r)\right)\right. \\
\left.-\underline{\theta}_{1}(r) \underline{g}(x, r) \frac{\partial^{2}}{\partial x^{2}} \underline{u}_{k}(x, t, r)\right] d t, \tilde{u}_{k+1}(x, 0, r, b)=0,
\end{gathered}
$$

where $k \geq 2$ and where $L^{-1}=\int_{0}^{t}() d$.$t is an inverse operator of the linear operator L=\frac{\partial}{\partial t}$. The approximate solution of Equation (16) can be obtained as follows:

$$
\tilde{u}(x, t, r, b)=\lim _{n \rightarrow \infty} b\left(\bar{u}_{k}(x, t, r)-\underline{u}_{k}(x, t, r)\right)+\underline{u}_{k}(x, t, r) .
$$

If we were to solve the fuzzy differential equation by means of a single parametric form of fuzzy numbers, then the HPM solution of upper and lower bounds of Equation (1) need to be analyzed and, hence, would demand more computational work. Therefore, the advantage of the parameter $b$ that deforms from 0 to 1 is the reduction of the computational and analysis work to obtain the solution of the equation. Subsequently, if $b=0$ in Equation (7), we can obtain the lower solution of Equation (1):

$$
\underline{u}(x, t, r)=\sum_{i=0}^{m-1} \underline{u}_{i}(x, t, r) .
$$


On the other hand, at $b=1$ we obtain the upper solution of Equation (1)

$$
\bar{u}(x, r)=\sum_{i=0}^{m-1} \bar{u}_{i}(x, t, r)
$$

and this is applicable for all the HPM analysis in double parametric form of fuzzy numbers.

\section{Double Parametric HPM Fuzzy Wave-Like Equation Analysis}

This section discusses the analysis of HPM fuzzy wave-like equations as in Equation (1) under fuzzy numbers in double parametric form. Based on the analysis in Sections 3 and 4, Equation (1) can be rewritten as follows:

$$
\begin{gathered}
H(x, p, r, b)=(1-p)\left\{b \left[\left(\frac{\partial^{2}}{\partial t^{2}} \bar{u}(x, t, r)-\frac{\partial^{2}}{\partial t^{2}} \bar{u}_{0}(x, t, r)\right)-\left(\frac{\partial^{2}}{\partial t^{2}} \underline{u}(x, t, r)-\right.\right.\right. \\
\left.\left.\left.\frac{\partial^{2}}{\partial t^{2}} \underline{u}_{0}(x, t, r)\right)\right]+\left(\frac{\partial^{2}}{\partial t^{2}} \underline{u}(x, t, r)-\frac{\partial^{2}}{\partial t^{2}} \underline{u}_{0}(x, t, r)\right)\right\}+p\left[b \left(\frac{\partial^{2}}{\partial t^{2}} \bar{u}(x, t, r)-\right.\right. \\
\left.\frac{\partial^{2}}{\partial t^{2}} \underline{u}(x, t, r)\right)+\frac{\partial^{2}}{\partial t^{2}} \underline{u}(x, t, r)+b\left(\bar{\eta}_{1}(r) \bar{h}(x, r) \frac{\partial^{2}}{\partial x^{2}} \bar{u}(x, t, r)-\right. \\
\left.\underline{\eta}_{1}(r) \underline{h}(x, r) \frac{\partial^{2}}{\partial x^{2}} \bar{u}(x, t, r)\right)+\underline{\eta}_{1}(r) \underline{h}(x, r) \frac{\partial^{2}}{\partial x^{2}} \bar{u}(x, t, r)-b\left(\bar{\eta}_{2}(r) \bar{w}(x, t, r)-\right. \\
\left.\left.\underline{\eta}_{2}(r) \underline{w}(x, t, r)\right)-\underline{\eta}_{2}(r) \underline{w}(x, t, r)\right]=0 .
\end{gathered}
$$

Then, the power series in $p$ representing the solution of Equation (20) can be written as Equation (13). The substitution of Equation (13) into Equation (20) yields the following:

$$
\begin{gathered}
H(x, p, r, b)=\left\{b \left[\left(\frac{\partial^{2}}{\partial t^{2}} \sum_{i=0}^{\infty} p^{i} \bar{u}_{i}(x, t, r)-\frac{\partial^{2}}{\partial t^{2}} \bar{u}_{0}(x, t, r)\right)-\left(\frac{\partial^{2}}{\partial t^{2}} \sum_{i=0}^{\infty} p^{i} \underline{u}_{i}(x, t, r)-\right.\right.\right. \\
\left.\left.\left.\frac{\partial^{2}}{\partial t^{2}} u_{0}(x, t, r)\right)\right]+\left(\frac{\partial^{2}}{\partial t^{2}} \sum_{i=0}^{\infty} p^{i} u_{i}(x, t, r)-\frac{\partial^{2}}{\partial t^{2}} u_{0}(x, t, r)\right)\right\}=p\left[-b\left(\frac{\partial^{2}}{\partial t^{2}} \bar{u}_{0}(x, t, r)-\right.\right. \\
\left.\frac{\partial^{2}}{\partial t^{2}} u_{0}(x, t, r)\right)+\frac{\partial^{2}}{\partial t^{2}} u_{0}(x, t, r)+b\left(\bar{\eta}_{1}(r) \bar{h}(x, r) \frac{\partial^{2}}{\partial x^{2}} \sum_{i=0}^{\infty} p^{i} \bar{u}_{i}(x, t, r)-\right. \\
\left.\underline{\eta}_{1}(r) \underline{h}_{0}(x, r) \frac{\partial^{2}}{\partial x^{2}} \sum_{i=0}^{\infty} p^{i} \underline{u}_{i}(x, t, r)\right)+\underline{\eta}_{1}(r) \underline{h}(x, r) \frac{\partial^{2}}{\partial x^{2}} \sum_{i=0}^{\infty} p^{i} \underline{u}_{i}(x, t, r)- \\
\left.b\left(\bar{\eta}_{2}(r) \bar{w}(x, t, r)-\underline{\eta}_{2}(r) \underline{w}(x, t, r)\right)+\underline{\eta}_{2}(r) \underline{w}(x, t, r)\right]=0 .
\end{gathered}
$$

From Section 6, the initial approximation function of Equation (21) is defined in the following form:

$$
\begin{gathered}
\tilde{u}_{0}(x, t, r, b)=b\left[\bar{u}_{0}(x, t, r)-\underline{u}_{0}(x, t, r)\right]+\underline{u}_{0}(x, t, r)=b\left[\bar{\eta}_{3}(r) \bar{d}(x, r)-\underline{\eta}_{3}(r) \underline{d}(x, r)\right] \\
+\underline{\eta}_{3}(r) \underline{d}(x, r)+\left(b\left[\bar{\eta}_{4}(r) \bar{q}(x, r)-\underline{\eta}_{4}(r) \underline{q}(x, r)\right]+\underline{\eta}_{4}(r) \underline{q}(x, r)\right) t .
\end{gathered}
$$


Again, the both sides with similar powers of $p$ are compared to obtain the solution such that the initial guess is Equation (22). Thus, the following recurrent relation is obtained:

$$
\begin{aligned}
& p^{1}: \tilde{u}_{1}(x, t, r, b)=\int_{0}^{T} \int_{0}^{T}\left[-b\left(\bar{\eta}_{1}(r) \bar{h}(x, r) \frac{\partial^{2}}{\partial x^{2}} \bar{u}_{0}(x, t, r)-\underline{\eta}_{1}(r) \underline{h}(x, r) \frac{\partial^{2}}{\partial x^{2}} \underline{u}_{0}(x, t, r)\right)\right. \\
& -\underline{\eta}_{1}(r) \underline{h}(x, r) \frac{\partial^{2}}{\partial x^{2}} \underline{u}_{0}(x, t, r) \\
& +b\left(\bar{\eta}_{2}(r) \bar{w}(x, t, r)-\underline{\eta}_{2}(r) \underline{w}(x, t, r)\right) \\
& \left.+\underline{\eta}_{2}(r) \underline{w}(x, t, r)\right] d t d t, \tilde{u}_{1}(x, 0, r, b)=0, \frac{\partial}{\partial t} \tilde{u}_{1}(x, 0, r, b) \\
& p^{2}: \tilde{u}_{2}(x, t, r, b)=\int_{0}^{T} \int_{0}^{T}\left[-b\left(\bar{\eta}_{1}(r) \bar{h}(x, r) \frac{\partial^{2}}{\partial x^{2}} \bar{u}_{1}(x, t, r)\right.\right. \\
& \left.-\underline{\eta}_{1}(r) \underline{h}(x, r) \frac{\partial^{2}}{\partial x^{2}} \underline{u}_{1}(x, t, r)\right) \\
& \left.-\underline{\eta}_{1}(r) \underline{h}(x, r) \frac{\partial^{2}}{\partial x^{2}} \underline{u}_{1}(x, t, r)\right] d t d t, \tilde{u}_{2}(x, 0, r, b) \\
& =0, \frac{\partial}{\partial t} \tilde{u}_{2}(x, 0, r, b)=0, \\
& p^{3}: \tilde{u}_{3}(x, t, r, b)=\int_{0}^{T} \int_{0}^{T}\left[-b\left(\bar{\eta}_{1}(r) \bar{h}(x, r) \frac{\partial^{2}}{\partial x^{2}} \bar{u}_{2}(x, t, r)\right.\right. \\
& \left.-\underline{\eta}_{1}(r) \underline{h}(x, r) \frac{\partial^{2}}{\partial x^{2}} \underline{u}_{2}(x, t, r)\right) \\
& \left.-\underline{\eta}_{1}(r) \underline{h}(x, r) \frac{\partial^{2}}{\partial x^{2}} \underline{u}_{2}(x, t, r)\right] d t d t, \tilde{u}_{3}(x, 0, r, b) \\
& =0, \frac{\partial}{\partial t} \tilde{u}_{3}(x, 0, r, b)=0 \text {, } \\
& p^{k+1}: \tilde{u}_{k}(x, t, r, b)=\int_{0}^{T} \int_{0}^{T}\left[-b\left(\bar{\eta}_{1}(r) \bar{h}(x, r) \frac{\partial^{2}}{\partial x^{2}} \bar{u}_{k}(x, t, r)\right.\right. \\
& \left.-\underline{\eta}_{1}(r) \underline{h}(x, r) \frac{\partial^{2}}{\partial x^{2}} \underline{u}_{k}(x, t, r)\right) \\
& \left.-\underline{\eta}_{1}(r) \underline{h}(x, r) \frac{\partial^{2}}{\partial x^{2}} \underline{u}_{2}(x, t, r)\right] d t d t, \tilde{u}_{k+1}(x, 0, r, b) \\
& =0, \frac{\partial}{\partial t} \tilde{u}_{k+1}(x, 0, r, b)=0 \text {, }
\end{aligned}
$$

where $k \geq 2$ and where $L^{-1}=\int_{0}^{T} \int_{0}^{T}() d t d$.$t is an inverse operator of the linear operator L=\frac{\partial^{2}}{\partial t^{2}}$. The approximate solution of Equation (23) can be obtained from Equation (17) in Section 6. As reasoned in the previous section, analyzing the solution of Equation (5) through single parametric fuzzy HPM for upper and lower bounds would demand more computational work. On the other hand, in double parametric form, the advantage of having the parameter changing from 0 to 1 is the reduction of the computational and analysis work. Therefore, if $b=0$ in Equation (7), the lower solution of Equation (5) can be obtained from Equation (18) and Equation (19) in Section 6, and this is applicable for all the HPM analysis in double parametric form of fuzzy numbers for all $r, b \in[0,1]$.

\section{Convergence Theorem}

Theorem 1. Let $[\tilde{X}]_{r}^{b}$ and $[\tilde{Y}]_{r}^{b}$ be the Banach spaces for each $r, b \in[0,1]$ in the crisp domain as given in [24]. Consider the nonlinear mapping $N:[\tilde{X}]_{r}^{b} \rightarrow[\tilde{Y}]_{r}^{b}$ such that

$$
\forall[\tilde{v}]_{r}^{b},[\tilde{\mathcal{V}}]_{r}^{b} \in[\tilde{X}]_{r}^{b}\left\|N\left([\tilde{\mathcal{V}}]_{r}^{b}\right)-N\left([\tilde{\mathcal{V}}]_{r}^{b}\right)\right\| \leq \varepsilon\left\|[\tilde{\mathcal{V}}]_{r}^{b}-[\tilde{\mathcal{V}}]_{r}^{b}\right\|, \varepsilon \in[0,1]
$$

where

$$
\begin{gathered}
{[\tilde{v}]_{r}^{b}=b\left([\bar{v}]_{r}-[\underline{v}]_{r}\right)-[\underline{v}]_{r} \text { and }} \\
{[\tilde{v}]_{r}^{b}=b\left([\bar{v}]_{r}-[v]_{r}\right)-[\underline{v}]_{r} .}
\end{gathered}
$$

Then, according to [24] there exists a unique fixed point $[\tilde{u}]_{r}^{b}$ such that

$$
N\left([\tilde{u}]_{r}^{b}\right)=[\tilde{u}]_{r}^{b}
$$


where $[\tilde{u}]_{r}^{b}=b\left([\bar{u}]_{r}-[\underline{u}]_{r}\right)-[\underline{u}]_{r}$.

Assume that the double parametric fuzzy HPM will generate the following form of sequence:

$$
\left[\tilde{\mathbb{V}}_{n}\right]_{r}^{b}=N\left(\left[\tilde{\mathbb{V}}_{n-1}\right]_{r}^{b}\right),\left[\tilde{\mathbb{V}}_{n-1}\right]_{r}^{b}=\sum_{1=0}^{n-1}\left[\tilde{u}_{i}\right]_{r}^{b}=\sum_{1=0}^{n-1}\left(b\left(\left[\bar{u}_{i}\right]_{r}-\left[\underline{u}_{i}\right]_{r}\right)-\left[\underline{u}_{i}\right]_{r}\right), n=1,2,3, \ldots,
$$

where

$$
\begin{gathered}
{\left[\tilde{\mathbb{V}}_{n}\right]_{r}^{b}=b\left(\left[\tilde{\mathbb{V}}_{n}\right]_{r}-\left[\tilde{\mathbb{V}}_{n}\right]_{r}\right)-\left[\tilde{\mathbb{V}}_{n}\right]_{r} \text { and }} \\
{\left[\tilde{\mathbb{V}}_{n-1}\right]_{r}^{b}=b\left(\left[\tilde{\mathbb{V}}_{n-1}\right]_{r}-\left[\tilde{\mathbb{V}}_{n-1}\right]_{r}\right)-\left[\tilde{\mathbb{V}}_{n-1}\right]_{r}}
\end{gathered}
$$

and suppose also that $\left[\tilde{\mathbb{V}}_{0}\right]_{r}^{b}=\left[\tilde{v}_{0}\right]_{r}^{b}=\left[\tilde{u}_{0}\right]_{r}^{b} \in B_{s}\left([\tilde{u}]_{r}^{b}\right)$ where $B_{s}\left([\tilde{u}]_{r}^{b}\right)=\left\{[\tilde{u}]_{r}^{b} \in X:\left\|[\tilde{u}]_{r}^{b}-[\tilde{u}]_{r}^{b}\right\|<s\right\}$. Then, for each $r, b \in[0,1]$ we have the following:

1. $\left\|\left[\tilde{\mathbb{V}}_{n}\right]_{r}^{b}-[\tilde{u}]_{r}^{b}\right\| \leq \varepsilon^{n}\left\|\left[\tilde{\mathbb{V}}_{0}\right]_{r}^{b}-[\tilde{u}]_{r}^{b}\right\|$

2. $\left[\tilde{\mathbb{V}}_{n}\right]_{r}^{b} \in B_{s}\left([\tilde{u}]_{r}^{b}\right)$, and

3. $\lim _{n \rightarrow \infty}\left[\tilde{\mathbb{V}}_{n}\right]_{r}^{b}=[\tilde{u}]_{r}^{b}$.

\section{Proof:}

1. Part (1) of the theorem can be proved using induction method [25,35]. For the double parametric solution of the heat-like equation in Equation (1), suppose that $[\tilde{u}]_{r}^{b}$ is the exact solution of Equation (1) defined in Equation (17) and let $\left[\widetilde{\mathbb{V}}_{0}\right]_{r}^{b}=\left[\tilde{v}_{0}\right]_{r}^{b}=\left[\tilde{u}_{0}\right]_{r}^{b}$ be the initial approximate function for double parametric fuzzy HPM. Then, for $n=1$ and by mathematical induction method, it follows that

$$
\left\|\left[\tilde{\mathbb{V}}_{1}\right]_{r}^{b}-[\tilde{u}]_{r}^{b}\right\|=\left\|N\left(\left[\tilde{\mathbb{V}}_{0}\right]_{r}^{b}\right)-N\left([\tilde{u}]_{r}^{b}\right)\right\| \leq \varepsilon\left\|\left[\tilde{\mathbb{V}}_{0}\right]_{r}^{b}-[\tilde{u}]_{r}^{b}\right\| .
$$

Let $\left\|\left[\tilde{\mathbb{V}}_{n-1}\right]_{r}^{b}-[\tilde{u}]_{r}^{b}\right\| \leq \varepsilon^{n-1}\left\|\left[\tilde{v}_{0}\right]_{r}^{b}-[\tilde{u}]_{r}^{b}\right\|$, then

$$
\begin{aligned}
\left\|\left[\tilde{\mathbb{V}}_{n}\right]_{r}^{b}-[\tilde{u}]_{r}^{b}\right\| \leq & \left\|N\left(\left[\tilde{\mathbb{V}}_{n-1}\right]_{r}^{b}\right)-N\left([\tilde{u}]_{r}^{b}\right)\right\| \leq \varepsilon\left\|\left[\tilde{\mathbb{V}}_{n-1}\right]_{r}^{b}-[\tilde{u}]_{r}^{b}\right\| \leq \varepsilon \varepsilon^{n-1}\left\|\left[\tilde{v}_{0}\right]_{r}^{b}-[\tilde{u}]_{r}^{b}\right\| \\
& \leq \varepsilon^{n}\left\|\left[\tilde{v}_{0}\right]_{r}^{b}-[\tilde{u}]_{r}^{b}\right\|, \text { (From induction hypothesis) }
\end{aligned}
$$

where

$$
\begin{gathered}
{\left[\tilde{\mathbb{V}}_{1}\right]_{r}^{b}=\left[\tilde{u}_{0}\right]_{r}^{b}+\left[\tilde{u}_{1}\right]_{r}^{b}} \\
{\left[\tilde{\mathbb{V}}_{n-1}\right]_{r}^{b}=\left[\tilde{u}_{0}\right]_{r}^{b}+\left[\tilde{u}_{1}\right]_{r}^{b}+\ldots+\left[\tilde{u}_{n-2}\right]_{r}^{b}+\left[\tilde{u}_{n-1}\right]_{r}^{b}}
\end{gathered}
$$

$\left[\tilde{\mathbb{V}}_{n}\right]_{r}^{b}=\left[\tilde{u}_{0}\right]_{r}^{b}+\left[\tilde{u}_{1}\right]_{r}^{b}+\ldots+\left[\tilde{u}_{n-2}\right]_{r}^{b}+\left[\tilde{u}_{n-1}\right]_{r}^{b}+\left[\tilde{u}_{n}\right]_{r}^{b}=\sum_{i=0}^{n}\left[\tilde{u}_{i}\right]_{r}^{b}$. This concludes the proof of part (1) of the theorem for the heat-like equation.

2. From part (1) of the theorem, we have

$$
\left\|\left[\tilde{\mathbb{V}}_{n}\right]_{r}^{b}-[\tilde{u}]_{r}^{b}\right\| \leq \varepsilon^{n}\left\|\left[\tilde{v}_{0}\right]_{r}^{b}-[\tilde{u}]_{r}^{b}\right\| \leq \varepsilon^{n} s \leq s
$$

This implies $\left[\tilde{\mathbb{V}}_{n}\right]_{r}^{b} \in B_{s}\left([\tilde{u}]_{r}^{b}\right)$, for every $r, b \in[0,1]$ and

3. From part (1) of the theorem, we also know that $\left\|\left[\tilde{\mathbb{V}}_{n}\right]_{r}^{b}-[\tilde{u}]_{r}^{b}\right\| \leq \varepsilon^{n}\left\|\left[\tilde{V}_{0}\right]_{r}^{b}-[\tilde{u}]_{r}^{b}\right\|$. 
Since $0<\varepsilon<1, \lim _{n \rightarrow \infty} \varepsilon^{n}=0$. So $\lim _{n \rightarrow \infty}\left\|\left[\tilde{\mathbb{V}}_{n}\right]_{r}^{b}-[\tilde{u}]_{r}^{b}\right\|=0$. This yields

$$
\lim _{n \rightarrow \infty}\left[\tilde{\mathbb{V}}_{n}\right]_{r}^{b}=[\tilde{u}]_{r}^{b}
$$

Thus, the series solution $\tilde{u}(s, r, b)$ in Equation (9) converges for each $r, b \in[0,1]$. This implies the series solution $\tilde{u}(x, t, r, b)$ in Equation (17) also converges for double parametric fuzzy HPM solution of Equation (1) for each $r, b \in[0,1]$. Note that the approximate solution for the fuzzy wave-like equation in Equation (23) can also be obtained from Equation (17) in Section 6.

\section{Findings and Discussion}

To test the potential of the developed approximation approach in Sections 6 and 7, we demonstrate how HPM can be used easily to obtain the approximate solution of one-dimensional fuzzy heat-like and fuzzy wave-like equations under double parametric fuzzy numbers.

Example 1. Given fuzzy heat-like equation with fuzzy initial condition [9]:

$$
\begin{gathered}
\tilde{u}_{t}(t, x)+\frac{\tilde{c}_{1}}{2} x^{2} \tilde{u}_{x x}(t, x)=\tilde{c}_{2}, \\
\tilde{u}(x, 0)=\tilde{c}_{3} x^{2}, 0<x<l, 0<t<T,
\end{gathered}
$$

where $\tilde{c}_{1}, \tilde{c}_{2}$, and $\tilde{c}_{3}$ are triangular fuzzy numbers with the fuzzy level sets $[r, 2-r]$. From [9], the exact solution of Equation (24) is given by

$$
\widetilde{U}(x, t)=\tilde{c}_{3} x^{2} e^{-\tilde{c}_{1} t}+\tilde{c}_{2} t .
$$

Based on Section 3, the double parametric form of Equation (24) is as follows:

$$
\begin{gathered}
b\left[\bar{u}_{t}(x, t, r)-\underline{u}_{t}(x, t, r)\right]+\underline{u}_{t}(x, t, r)=b\left[\frac{(2-r) x^{2}}{2} \bar{u}_{x x}(x, t, r)-\frac{r x^{2}}{2} \underline{u}_{x x}(x, t, r)\right]+ \\
\frac{r x^{2}}{2} \underline{u}_{x x}(x, t, r)=b[(2-2 r) t]+r t . \\
b[\bar{u}(x, 0, r)-\underline{u}(x, 0, r)]+\underline{u}(x, 0, r)=b\left[(2-2 r) x^{2}\right]+r x^{2}
\end{gathered}
$$

where $b \in[0,1]$ is a free parameter. Moreover, the exact solution of Equation (24) can be written in a fuzzy double parametric:

$$
b[\bar{U}(x, t, r)-\underline{U}(x, t, r)]+\underline{U}(x, t, r)=b\left[(2-r) x^{2} e^{-(2-r) t}-r x^{2} e^{-r t}\right]+r x^{2} e^{-r t}+b[(2-2 r) t]+r t .
$$

By setting $b=0$, we obtain the lower bound exact solution of Equation (24) such that where $\underline{U}(x, t, r)=r x^{2} e^{-r t}+r t$, and if $b=1$ then the upper exact solution of Equation (24) is 
$\bar{U}(x, t, r)=(2-r) x^{2} e^{-(2-r) t}+(2-r) t$. According to the analysis of HPM in Sections 4 and 5 we yield the following:

$$
\begin{aligned}
& p^{0}:\left\{b\left(\bar{u}_{0}(x, t, r)-\underline{u}_{0}(x, t, r)\right)+u_{0}(x, t, r)=b\left[(2-2 r) x^{2}\right]+r x^{2}\right. \\
& p^{1}:\left\{\begin{array}{c}
b\left(\bar{u}_{1}(x, t, r)-\underline{u}_{1}(x, t, r)\right)+\underline{u}_{1}(x, t, r)= \\
\int_{0}^{t}\left[b\left(\frac{(2-r) x^{2}}{2} \frac{\partial^{2} \bar{u}_{0}(x, t, r)}{\partial x^{2}}-\frac{r x^{2}}{2} \frac{\partial^{2} \bar{u}_{0}(x, t ; r)}{\partial x^{2}}\right)+\frac{r x^{2}}{2} \frac{\partial^{2} \bar{u}_{0}(x, t ; r)}{\partial x^{2}}+(b[(2-2 r) t]+r t)\right] d t, \\
b\left[\bar{u}_{1}(x, 0, r)-\underline{u}_{1}(x, 0, r)\right]+\underline{u}_{1}(x, 0, r)=0,
\end{array}\right. \\
& p^{2}:\left\{\begin{array}{c}
b\left(\bar{u}_{2}(x, t, r)-\underline{u}_{2}(x, t, r)\right)+\underline{u}_{2}(x, t, r)= \\
\int_{0}^{t}\left[b\left(\frac{(2-r) x^{2}}{2} \frac{\partial^{2} \bar{u}_{1}(x, t ; r)}{\partial x^{2}}-\frac{r x^{2}}{2} \frac{\partial^{2} \bar{u}_{1}(x, t, r)}{\partial x^{2}}\right)+\frac{r x^{2}}{2} \frac{\partial^{2} \bar{u}_{1}(x, t ; r)}{\partial x^{2}}\right] d t \\
b\left[\bar{u}_{2}(x, 0, r)-\underline{u}_{2}(x, 0, r)\right]+\underline{u}_{2}(x, 0, r)=0
\end{array}\right. \\
& p^{k+1}:\left\{\begin{array}{c}
b\left(\bar{u}_{k+1}(x, t, r)-\underline{u}_{k+1}(x, t, r)\right)+\underline{u}_{k+1}(x, t, r)= \\
\int_{0}^{t}\left[b\left(\frac{(2-r) x^{2}}{2} \frac{\partial^{2} \bar{u}_{k+1}(x, t ; r)}{\partial x^{2}}-\frac{r x^{2}}{2} \frac{\partial^{2} \bar{u}_{k+1}(x, t ; r)}{\partial x^{2}}\right)+\frac{r x^{2}}{2} \frac{\partial^{2} \bar{u}_{k+1}(x, t ; r)}{\partial x^{2}}\right] d t \\
b\left[\bar{u}_{k+1}(x, 0, r)-\underline{u}_{k+1}(x, 0, r)\right]+\underline{u}_{k+1}(x, 0, r)=0,
\end{array}\right.
\end{aligned}
$$

Next, define the third and fifth order double parametric fuzzy HPM approximate series solution of Equation (24) as follows:

$$
\tilde{u}(x, t, r, b)_{3,5}=\sum_{i=0}^{3,5} b\left(\bar{u}_{i}(x, t, r)-\underline{u}_{i}(x, t, r)\right)+\underline{u}_{i}(x, t, r)
$$

By using Mathematica 12 software code, we obtain the corresponding third and fifth order series solution from Equation (28) as follows:

$$
\begin{aligned}
\tilde{u}(x, t, r, b)_{3}= & \left\{0.0625, b(2-2 r) x^{2}+r x^{2}+4 b^{3} t^{2} x^{2}+6 b^{2} r t^{2} x^{2}-12 b^{3} r t^{2} x^{2}+3 b r^{2} t^{2} x^{2}\right. \\
& -12 b^{2} r^{2} t^{2} x^{2}+12 b^{3} r^{2} t^{2} x^{2}+\frac{1}{2} r^{3} t^{2} x^{2}-3 b r^{3} t^{2} x^{2}+6 b^{2} r^{3} t^{2} x^{2} \\
& -4 b^{3} r^{3} t^{2} x^{2}-\frac{8}{3} b^{4} t^{3} x^{2}-\frac{16}{3} b^{3} r t^{3} x^{2}+\frac{32}{3} b^{4} r t^{3} x^{2}-4 b^{2} r^{2} t^{3} x^{2} \\
& +16 b^{3} r^{2} t^{3} x^{2}-16 b^{4} r^{2} t^{3} x^{2}-\frac{4}{3} b r^{3} t^{3} x^{2}+8 b^{2} r^{3} t^{3} x^{2}-16 b^{3} r^{3} t^{3} x^{2} \\
& +\frac{32}{3} b^{4} r^{3} t^{3} x^{2}-\frac{1}{6} r^{4} t^{3} x^{2}+\frac{4}{3} b r^{4} t^{3} x^{2}-4 b^{2} r^{4} t^{3} x^{2}+\frac{16}{3} b^{3} r^{4} t^{3} x^{2} \\
& -\frac{8}{3} b^{4} r^{4} t^{3} x^{2} \\
& +t\left(b(2-2 r)+r-\frac{1}{2} r(2 b(2-2 r)+2 r) x^{2}\right. \\
& \left.\left.-b\left(\frac{1}{2}(2-r)(2 b(2-2 r)+2 r) x^{2}-\frac{1}{2} r(2 b(2-2 r)+2 r) x^{2}\right)\right)\right\}
\end{aligned}
$$




$$
\begin{aligned}
& \tilde{u}(x, t, r, b)_{5}=\left\{0.1875, b(2-2 r) x^{2}+r x^{2}+4 b^{3} t^{2} x^{2}+6 b^{2} r t^{2} x^{2}-12 b^{3} r t^{2} x^{2}+3 b r^{2} t^{2} x^{2}\right. \\
& -12 b^{2} r^{2} t^{2} x^{2}+12 b^{3} r^{2} t^{2} x^{2}+\frac{1}{2} r^{3} t^{2} x^{2}-3 b r^{3} t^{2} x^{2}+6 b^{2} r^{3} t^{2} x^{2} \\
& -4 b^{3} r^{3} t^{2} x^{2}-\frac{8}{3} b^{4} t^{3} x^{2}-\frac{16}{3} b^{3} r t^{3} x^{2}+\frac{32}{3} b^{4} r t^{3} x^{2}-4 b^{2} r^{2} t^{3} x^{2} \\
& +16 b^{3} r^{2} t^{3} x^{2}-16 b^{4} r^{2} t^{3} x^{2}-\frac{4}{3} b r^{3} t^{3} x^{2}+8 b^{2} r^{3} t^{3} x^{2}-16 b^{3} r^{3} t^{3} x^{2} \\
& +\frac{32}{3} b^{4} r^{3} t^{3} x^{2}-\frac{1}{6} r^{4} t^{3} x^{2}+\frac{4}{3} b r^{4} t^{3} x^{2}-4 b^{2} r^{4} t^{3} x^{2}+\frac{16}{3} b^{3} r^{4} t^{3} x^{2} \\
& -\frac{8}{3} b^{4} r^{4} t^{3} x^{2}+\frac{4}{3} b^{5} t^{4} x^{2}+\frac{10}{3} b^{4} r t^{4} x^{2}-\frac{20}{3} b^{5} r t^{4} x^{2}+\frac{10}{3} b^{3} r^{2} t^{4} x^{2} \\
& -\frac{40}{3} b^{4} r^{2} t^{4} x^{2}+\frac{40}{3} b^{5} r^{2} t^{4} x^{2}+\frac{5}{3} b^{2} r^{3} t^{4} x^{2}-10 b^{3} r^{3} t^{4} x^{2}+20 b^{4} r^{3} t^{4} x^{2} \\
& -\frac{40}{3} b^{5} r^{3} t^{4} x^{2}+\frac{5}{12} b r^{4} t^{4} x^{2}-\frac{10}{3} b^{2} r^{4} t^{4} x^{2}+10 b^{3} r^{4} t^{4} x^{2}-\frac{40}{3} b^{4} r^{4} t^{4} x^{2} \\
& +\frac{20}{3} b^{5} r^{4} t^{4} x^{2}+\frac{1}{24} r^{5} t^{4} x^{2}-\frac{5}{12} b r^{5} t^{4} x^{2}+\frac{5}{3} b^{2} r^{5} t^{4} x^{2}-\frac{10}{3} b^{3} r^{5} t^{4} x^{2} \\
& +\frac{10}{3} b^{4} r^{5} t^{4} x^{2}-\frac{4}{3} b^{5} r^{5} t^{4} x^{2}-\frac{8}{15} b^{6} t^{5} x^{2}-\frac{8}{5} b^{5} r t^{5} x^{2}+\frac{16}{5} b^{6} r t^{5} x^{2} \\
& -2 b^{4} r^{2} t^{5} x^{2}+8 b^{5} r^{2} t^{5} x^{2}-8 b^{6} r^{2} t^{5} x^{2}-\frac{4}{3} b^{3} r^{3} t^{5} x^{2}+8 b^{4} r^{3} t^{5} x^{2} \\
& -16 b^{5} r^{3} t^{5} x^{2}+\frac{32}{3} b^{6} r^{3} t^{5} x^{2}-\frac{1}{2} b^{2} r^{4} t^{5} x^{2}+4 b^{3} r^{4} t^{5} x^{2}-12 b^{4} r^{4} t^{5} x^{2} \\
& +16 b^{5} r^{4} t^{5} x^{2}-8 b^{6} r^{4} t^{5} x^{2}-\frac{1}{10} b r^{5} t^{5} x^{2}+b^{2} r^{5} t^{5} x^{2}-4 b^{3} r^{5} t^{5} x^{2} \\
& +8 b^{4} r^{5} t^{5} x^{2}-8 b^{5} r^{5} t^{5} x^{2}+\frac{16}{5} b^{6} r^{5} t^{5} x^{2}-\frac{1}{120} r^{6} t^{5} x^{2}+\frac{1}{10} b r^{6} t^{5} x^{2} \\
& -\frac{1}{2} b^{2} r^{6} t^{5} x^{2}+\frac{4}{3} b^{3} r^{6} t^{5} x^{2}-2 b^{4} r^{6} t^{5} x^{2}+\frac{8}{5} b^{5} r^{6} t^{5} x^{2}-\frac{8}{15} b^{6} r^{6} t^{5} x^{2} \\
& +t\left(b(2-2 r)+r-\frac{1}{2} r(2 b(2-2 r)+2 r) x^{2}\right. \\
& \left.\left.-b\left(\frac{1}{2}(2-r)(2 b(2-2 r)+2 r) x^{2}-\frac{1}{2} r(2 b(2-2 r)+2 r) x^{2}\right)\right)\right\} .
\end{aligned}
$$

From the above solutions the third and fifth order HPM double parametric it involve extra parameters $r$ and $b$ with two independent variables $t$ and $x$ but even with these parameters it is found that the third order solution $\tilde{u}(x, t, r, b)_{3}$ is obtained within $0.0625 \mathrm{~s}$ while the fifth order solution $\tilde{u}(x, t, r, b)_{5}$ is obtained within $0.1875 \mathrm{~s}$. Clearly, this double parametric fuzzy number approximation approach based on HPM is a fast method with respect to CPU time consumption.

Moreover, from Section 8 the convergence of double parametric fuzzy HPM solution of Equation (24) can be shown by setting $b=1$ and $r=0$ in Equation (28) to obtain the following:

$$
\begin{gathered}
\tilde{u}_{0}(x, t, 0,1)=2 x^{2}, \\
\tilde{u}_{1}(x, t, 0,1)=t\left(2-4 x^{2}\right), \\
\tilde{u}_{2}(x, t, 0,1)=4 t^{2} x^{2}, \\
\tilde{u}_{3}(x, t, 0,1)=-\frac{8}{3} t^{3} x^{2}, \\
\tilde{u}_{4}(x, t, 0,1)=\frac{4}{3} t^{4} x^{2}, \\
\tilde{u}_{5}(x, t, 0,1)=-\frac{8}{15} t^{5} x^{2}, \\
\tilde{u}_{6}(x, t, 0,1)=\frac{8}{45} t^{6} x^{2}, \\
\cdots \\
\tilde{u}(x, t, 0,1)_{n}=\left(2 t+x^{2} \sum_{n=0}^{\infty} \frac{(-1)^{n} 2^{n+1} t^{n}}{n !}\right)
\end{gathered}
$$

From Equation (27), the exact solution of Equation (24) for $b=1$ and $r=0$ is $\tilde{U}(x, t, 0,1)=$ $2 t+2 \mathrm{e}^{-2 t} x^{2}$. From the convergence theorem in Section 8 , we have the following relation:

$$
\lim _{n \rightarrow \infty}\left\|\left(2 t+x^{2} \sum_{n=0}^{\infty} \frac{(-1)^{n} 2^{n+1} t^{n}}{n !}\right)-\left(2 t+2 \mathrm{e}^{-2 t} x^{2}\right)\right\|=0 \quad \rightarrow \lim _{n \rightarrow \infty}\left(2 t+x^{2} \sum_{n=0}^{\infty} \frac{(-1)^{n} 2^{n+1} t^{n}}{n !}\right)=\left(2 t+2 \mathrm{e}^{-2 t} x^{2}\right)
$$

where $\lim _{n \rightarrow \infty}\left(\sum_{n=0}^{\infty} \frac{(-1)^{n} 2^{n+1} t^{n}}{n !}\right)=2 \mathrm{e}^{-2 t}$. Thus, in the same manner the double parametric fuzzy HPM solution converges to the exact solution of Equation (24) for each $r, b \in[0,1]$. The error analysis of double parametric HPM series solution of Equation (24) for all $r, b \in[0,1]$ is defined by the absolute errors between the exact solution (27) and the approximate solution (28) in the following form:

$$
\tilde{E}(x, t, r, b)_{3,5}=\left|\widetilde{U}(x, t, r, b)-\tilde{u}(x, t, r, b)_{3,5}\right| .
$$


The numerical results in double parametric form that obtained by third and fifth order HPM are compared with the exact solutions of Equation (24) for different values $r, b \in[0,1]$ when $t, x=0.1$ are displayed in Tables 1-6. Additionally, the double parametric form exact and approximate solutions of Equation (24) for different values $r, b \in[0,1]$ when $t, x=0.1$ are also shown graphically in Figure 2.

Table 1. Solution and accuracy of third and fifth order double parametric HPM solutions for Equation (24) at $t, x=0.1$ and $b=0$ for all $r \in[0,1]$.

\begin{tabular}{cccccc}
\hline $\boldsymbol{r}$ & $\boldsymbol{u}(0.1,0.1,0, \boldsymbol{r})_{3}$ & $\boldsymbol{u}(0.1,0.1,0, \boldsymbol{r})_{5}$ & $\boldsymbol{U}(0.1,0.1,0, \boldsymbol{r})$ & $\boldsymbol{E}(0.1,0.1,0, \boldsymbol{r})_{3}$ & $\boldsymbol{E}(0.1,0.1,0, \boldsymbol{r})_{5}$ \\
\hline 0 & 0 & 0 & 0 & 0 & 0 \\
0.2 & 0.021960397346613340 & 0.021960397346613515 & 0.021960397333333336 & $1.3280 \times 10^{-11}$ & $1.7694 \times 10^{-16}$ \\
0.4 & 0.043843157756586676 & 0.043843157756609304 & 0.043843157333333334 & $4.2327 \times 10^{-10}$ & $2.2627 \times 10^{-14}$ \\
0.6 & 0.065650587201120000 & 0.065650587201505500 & 0.06565058400000001 & $3.20150 \times 10^{-9}$ & $3.8549 \times 10^{-13}$ \\
0.8 & 0.087384930768213350 & 0.087384930771093100 & 0.08738491733333335 & $1.34377 \times 10^{-8}$ & $2.8797 \times 10^{-12}$ \\
1 & 0.109048374166666680 & 0.109048374180359600 & 0.10904833333333334 & $4.08470 \times 10^{-8}$ & $1.3692 \times 10^{-11}$ \\
\hline
\end{tabular}

Table 2. Solution and accuracy of third and fifth order double parametric fuzzy HPM solutions for Equation (24) at $t, x=0.1$ and $b=0.2$ for all $r \in[0,1]$.

\begin{tabular}{cccccc}
\hline $\boldsymbol{r}$ & $\boldsymbol{u}(0.1,0.1,0.2, \boldsymbol{r})_{3}$ & $\boldsymbol{u}(0.1,0.1,0.2, \boldsymbol{r})_{5}$ & $\boldsymbol{U}(0.1,0.1,0.2, \boldsymbol{r})$ & $\boldsymbol{E}(0.1,0.1,0.2, \boldsymbol{r})_{3}$ & $\begin{array}{l}\boldsymbol{E} 0.1,0.1,0.2, \boldsymbol{r}_{5} \\
-\end{array}$ \\
\hline 0 & 0.043843157756586676 & 0.043274923012311936 & 0.04384315733333334 & 0.00056823432 & 0.00083508474 \\
0.2 & 0.056936510107441300 & 0.056575290638371400 & 0.05693650853973334 & 0.00036121790 & 0.00053303861 \\
0.4 & 0.070003231996391370 & 0.069801386329979310 & 0.07000322757973333 & 0.00020184124 & 0.00029908080 \\
0.6 & 0.083043803167839120 & 0.082954672820321070 & 0.08304379276373333 & 0.00008911994 & 0.00008913034 \\
0.8 & 0.096058695709559830 & 0.096036553664995650 & 0.09605867410773335 & 0.00002212044 & 0.00002214204 \\
1 & 0.109048374166666670 & 0.109048374180359600 & 0.10904833333333334 & $4.08470 \times 10^{-8}$ & $1.3692 \times 10^{-11}$ \\
\hline
\end{tabular}

Table 3. Solution and accuracy of third and fifth order double parametric fuzzy HPM solutions for Equation (24) at $t, x=0.1$ and $b=0.4$ for all $r \in[0,1]$.

\begin{tabular}{cccccc}
\hline $\boldsymbol{r}$ & $\boldsymbol{u}(0.1,0.1,0.4, \boldsymbol{r})_{3}$ & $\boldsymbol{u}(0.1,0.1,0.4, \boldsymbol{r})_{5}$ & $\boldsymbol{U}(0.1,0.1,0.4, \boldsymbol{r})$ & $\begin{array}{c}\boldsymbol{E}(0.1,0.1,0.4, \boldsymbol{r})_{3} \\
-\end{array}$ & $\begin{array}{c}\boldsymbol{E}(0.1,0.1,0.4, \boldsymbol{r})_{5} \\
-\end{array}$ \\
\hline 0 & 0.08738493076821334 & 0.08654984602462387 & 0.08738491733333335 & 0.0008350713 \\
0.2 & 0.09172322254714928 & 0.09119018393012929 & 0.09172320541440002 & 0.0005330214 & 0.00083508474 \\
0.4 & 0.09605869570955981 & 0.09575961490334933 & 0.09605867410773335 & 0.0002990592 & 0.00029908080 \\
0.6 & 0.10039136736816764 & 0.10025875843913660 & 0.10039134041173334 & 0.0001325819 & 0.00013260892 \\
0.8 & 0.10472125454396425 & 0.10468817655889823 & 0.1047212212224 & 0.0000330446 & 0.00003307798 \\
1 & 0.10904837416666667 & 0.10904837418035960 & 0.10904833333333334 & $4.08470 \times 10^{-8}$ & $1.3692 \times 10^{-11}$ \\
\hline
\end{tabular}

Table 4. Solution and accuracy of third and fifth order double parametric fuzzy HPM solutions for Equation (24) at $t, x=0.1$ and $b=0.6$ for all $r \in[0,1]$.

\begin{tabular}{cccccc}
\hline $\boldsymbol{r}$ & $\overline{\boldsymbol{u}}(0.1,0.1,0.6, \boldsymbol{r})_{3}$ & $\overline{\boldsymbol{u}}(0.1,0.1,0.6, \boldsymbol{r})_{5}$ & $\overline{\boldsymbol{U}}(0.1,0.1,0.6, \boldsymbol{r})$ & $\overline{\boldsymbol{E}}(0.1,0.1,0.6, \boldsymbol{r})_{3}$ & $\overline{\boldsymbol{E}}(0.1,0.1,0.6, \boldsymbol{r})_{5}$ \\
\hline 0 & 0.13064304519168002 & 0.12982476903693577 & 0.130642944 & 0.00081817496 & 0.00081827615 \\
0.2 & 0.12632951255163880 & 0.12580507722188716 & 0.12632942706773334 & 0.00052434984 & 0.00052443532 \\
0.4 & 0.12201329565378458 & 0.12171784347671935 & 0.12201322386773333 & 0.00029538039 & 0.00029545217 \\
0.6 & 0.11769437801800796 & 0.12171784347671935 & 0.11769431811840005 & 0.00013147406 & 0.00013153396 \\
0.8 & 0.11337274307517177 & 0.11333979945280077 & 0.11337269343573336 & 0.00003289398 & 0.00003294362 \\
1 & 0.10904837416666668 & 0.10904837418035960 & 0.10904833333333334 & $4.08470 \times 10^{-8}$ & $1.3692 \times 10^{-11}$ \\
\hline
\end{tabular}

Table 5. Solution and accuracy of third and fifth order double parametric fuzzy HPM solutions for Equation (24) at $t, x=0.1$ and $b=0.8$ for all $r \in[0,1]$.

\begin{tabular}{cccccc}
\hline $\boldsymbol{r}$ & $\overline{\boldsymbol{u}}(0.1,0.1,0.8, \boldsymbol{r})_{3}$ & $\overline{\boldsymbol{u}}(0.1,0.1,0.8, \boldsymbol{r})_{5}$ & $\overline{\boldsymbol{U}}(0.1,0.1,0.8, \boldsymbol{r})$ & $\overline{\boldsymbol{E}}(0.1,0.1,0.8, \boldsymbol{r})_{3}$ & $\overline{\boldsymbol{E}}(0.1,0.1,0.8, \boldsymbol{r})_{5}$ \\
\hline 0 & 0.17363430025898668 & 0.17309969204924774 & 0.17363387733333335 & 0.00053418528 & 0.00053460820 \\
0.2 & 0.16076398028960376 & 0.16041997051364504 & 0.16076369317973338 & 0.00034372266 & 0.00034400977 \\
0.4 & 0.14787065968461000 & 0.14767607205008937 & 0.14787047109973328 & 0.00019439904 & 0.00019458763 \\
0.6 & 0.13495390996543170 & 0.13486692967676772 & 0.13495379084373335 & 0.00008686116 & 0.00008698028 \\
0.8 & 0.12201329565378456 & 0.12199142234670335 & 0.12201322386773333 & 0.00002180152 & 0.00002187330 \\
1 & 0.10904837416666667 & 0.10904837418035960 & 0.10904833333333333 & $4.08470 \times 10^{-8}$ & $1.3692 \times 10^{-11}$ \\
\hline
\end{tabular}


Table 6. Solution and accuracy of third and fifth order double parametric fuzzy HPM solutions for Equation (24) at $t, x=0.1$ and $b=1$ for all $r \in[0,1]$.

\begin{tabular}{cccccc}
\hline $\boldsymbol{r}$ & $\overline{\boldsymbol{u}}(0.1,0.1,1, \boldsymbol{r})_{3}$ & $\overline{\boldsymbol{u}}(0.1,0.1,1, \boldsymbol{r})_{5}$ & $\overline{\boldsymbol{U}}(0.1,0.1,1, \boldsymbol{r})$ & $\overline{\boldsymbol{E}}(0.1,0.1,1, \boldsymbol{r})_{3}$ & $\overline{\boldsymbol{E}}(0.1,0.1,1, \boldsymbol{r})_{5}$ \\
\hline 0 & 0.21637461333333335 & 0.21637461506155964 & 0.216373333333333333 & 0.000001281728 & $1.72822 \times 10^{-9}$ \\
0.2 & 0.19503486297648004 & 0.19503486380540294 & 0.19503410400000004 & $7.59805 \times 10^{-7}$ & $8.2892 \times 10^{-10}$ \\
0.4 & 0.17363430025898670 & 0.17363430062345940 & 0.17363387733333335 & $4.23290 \times 10^{-7}$ & $3.6447 \times 10^{-10}$ \\
0.6 & 0.15217101515205333 & 0.15217101529558330 & 0.15217079733333333 & $2.17962 \times 10^{-7}$ & $1.4352 \times 10^{-10}$ \\
0.8 & 0.13064304519168002 & 0.13064304524060590 & 0.13064294399999998 & $1.01240 \times 10^{-7}$ & $4.8925 \times 10^{-11}$ \\
1 & 0.10904837416666666 & 0.10904837418035960 & 0.10904833333333334 & $4.08470 \times 10^{-8}$ & $1.3692 \times 10^{-11}$ \\
\hline
\end{tabular}

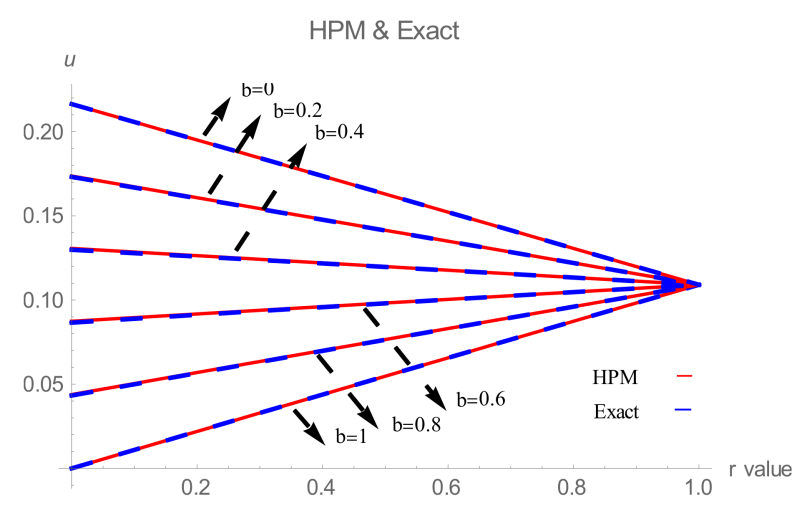

Figure 2. Comparison of the exact and fifth-order double parametric fuzzy HPM solutions of Equation

(24) at $x, t=0.1$ for different values of $r, b \in[0,1]$.

Clearly from Tables 1-6 and Figure 2, all the solutions for $b=0,0.2,0.4$ give the lower fuzzy solutions of Equation (24) while the solutions for $b=0.6,0.8,1$ give the upper fuzzy solution of Equation (24) for all $r \in[0,1]$ satisfy the properties of triangular fuzzy number. Also we also conclude that the fifth order double parametric fuzzy HPM series solution converges with higher accuracy to the exact solution of Equation (24).
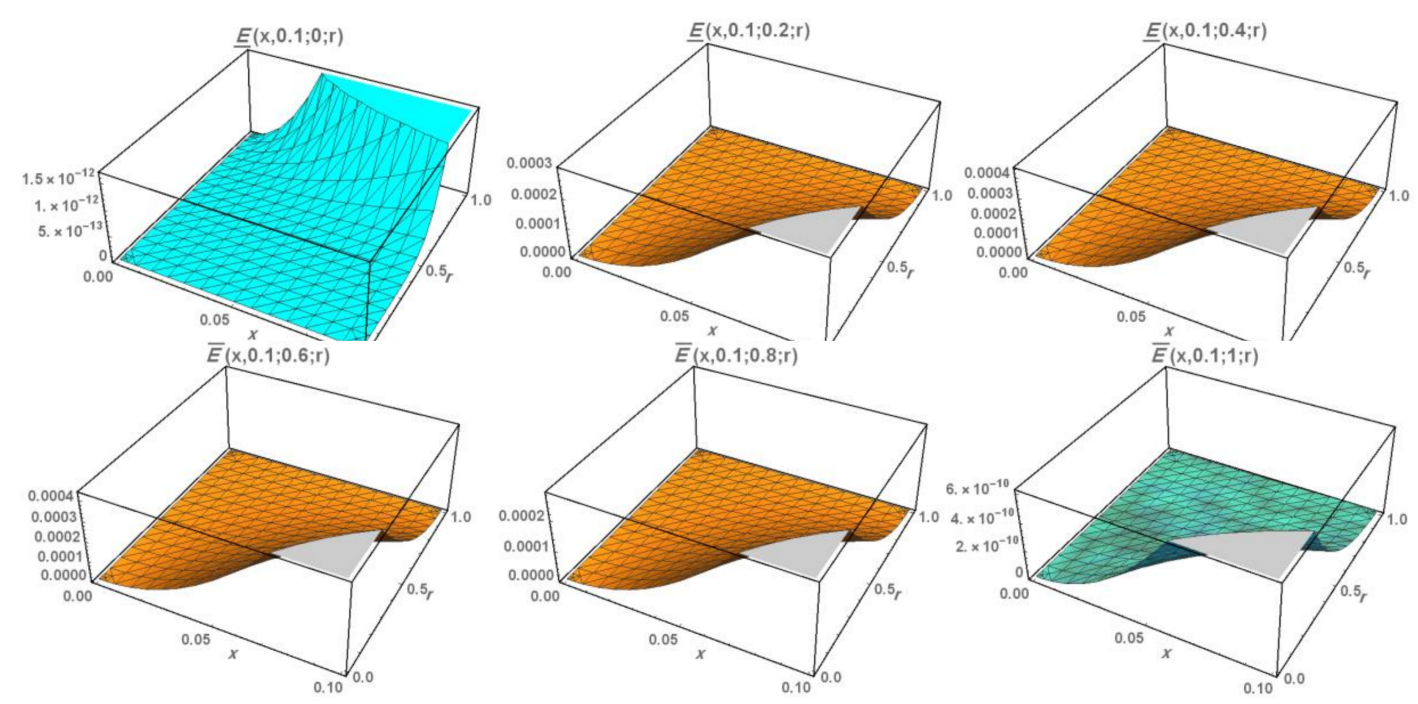

Figure 3. Accuracy of fifth-order HPM solution at $t=0.1, b=0,0.2,0.4,0.6,0.8,1$ for all $x \in[0,0.1]$ and $r \in[0,1]$.

Figure 3 is used to display the accuracy of fifth order double parametric fuzzy HPM upper and lower solutions of Equation (24) in three-dimensional shape for $t=0.1$ over the interval $x \in[0,0.1]$ and different values of $b=0,0.2,0.4,0.6,0.8,1$ for all $r \in[0,1]$. Moreover, the fifth order double 
parametric HPM approximate solutions of Equation (24) for all $x \in[0,0.1]$ and $r \in[0,1]$ for different values of $b=0,0.2,0.4,0.6,0.8,1$ when $t=0.1$ are illustrated graphically in Figures 4-6.

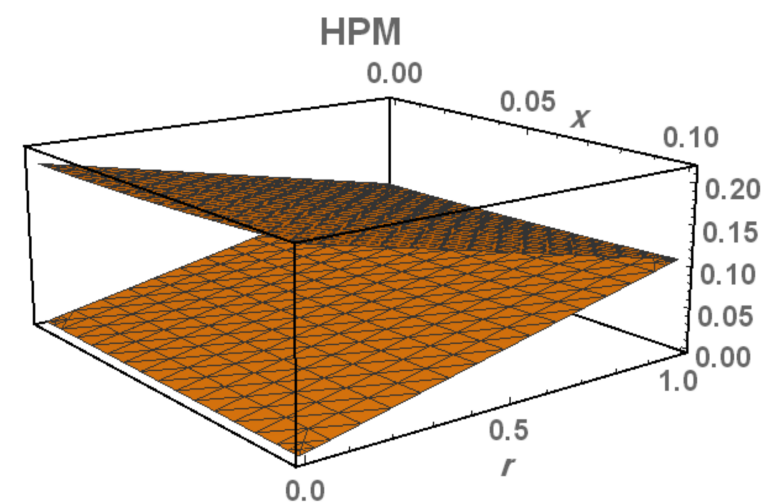

Figure 4. Solution of fifth-order double parametric fuzzy HPM at $t=0.1, b=0,1$ for all $x \in[0,0.1]$ and $r \in[0,1]$.

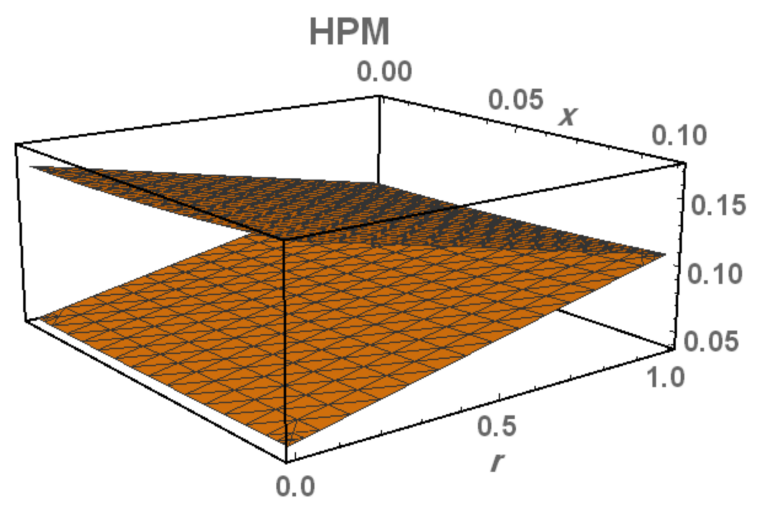

Figure 5. Solution using the fifth-order double parametric fuzzy HPM at $t=0.1, b=0.2,0.8$ for all $x \in[0,0.1]$ and $r \in[0,1]$.

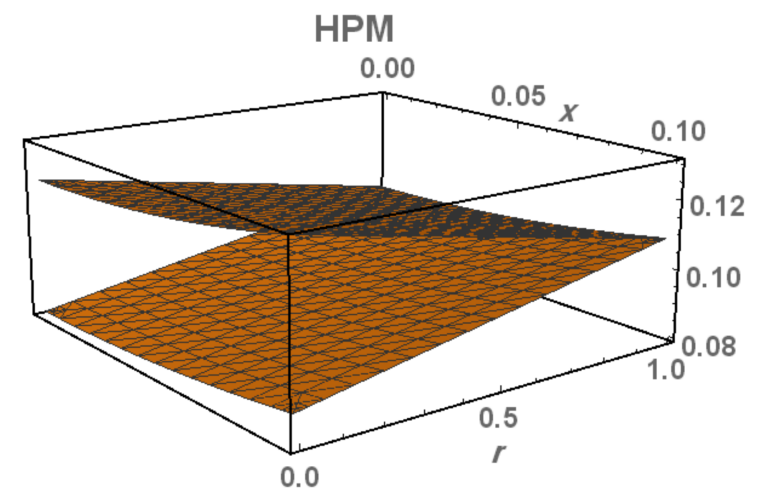

Figure 6. Solution using fifth-order double parametric fuzzy HPM at $t=0.1, b=0.4,0.6$ for all $x \in[0,0.1]$ and $r \in[0,1]$.

Figures 4-6 shows that the fifth order double parametric fuzzy HPM solutions of Equation (24) for all $x \in[0,0.1]$ and $r \in[0,1]$ of different values $b=0,0.2,0.4,0.6,0.8,1$ when $t=0.1$ are meet the properties of triangular fuzzy numbers.

Example 2: Given fuzzy wave-like equation with fuzzy initial conditions [18]: 


$$
\begin{gathered}
\tilde{u}_{t t}(x, t)+\frac{x^{2}}{2} \tilde{u}_{x x}(x, t)=\tilde{a}_{1} x t, \\
\tilde{u}(x, 0)=\tilde{a}_{2} x, \tilde{u}_{t}(x, 0)=\tilde{a}_{3} x^{2} 0<x<1,0<t<\pi
\end{gathered}
$$

where triangular fuzzy numbers in Equation (31) are $\tilde{a}_{1}=[r+1,3-r], \tilde{a}_{2}=[r-1,1-r]$ and $\tilde{a}_{3}=$ $[r, 2-r]$. According to [16], the exact solution of Equation (31) is given by

$$
\widetilde{U}(x, t)=\tilde{a}_{2} x+\tilde{a}_{3} x^{2} \sin (t)+\tilde{a}_{1} \frac{x t^{3}}{6} .
$$

Similar to the previous example, the fuzzy double parametric form of Equation (31) is given by the following:

$$
\begin{gathered}
b\left[\bar{u}_{t t}(x, t, r)-\underline{u}_{t t}(x, t, r)\right]+\underline{u}_{t t}(x, t, r)=b\left[\frac{x^{2}}{2} \bar{u}_{x x}(x, t, r)-\frac{x^{2}}{2} \underline{u}_{x x}(x, t, r)\right]+ \\
\frac{x^{2}}{2} \underline{u}_{x x}(x, t, r)=b[(2-2 r) t x]+(r+1) x t, \\
b[\bar{u}(x, 0, r)-\underline{u}(x, 0, r)]+\underline{u}(x, 0, r)=b[(2-2 r) x]+(r-1) x, \\
b\left[\bar{u}_{t}(x, 0, r)-\underline{u}_{t}(x, 0, r)\right]+\underline{u}_{t}(x, 0, r)=b\left[(2-2 r) x^{2}\right]+r x^{2},
\end{gathered}
$$

where $b \in[0,1]$ is a free parameter. From Section 3, the exact solution of Equation (31) in fuzzy double parametric form is as follows:

$$
\begin{aligned}
& b[\bar{U}(x, t, r)-\underline{U}(x, t, r)]+\underline{U}(x, t, r)=b[(2-2 r) x]+(r-1) x+ \\
& b\left[(2-2 r) x^{2} \sin (t)\right]+r x^{2} \sin (t)+b\left[(2-2 r) \frac{x t^{3}}{6}\right]+(r+1) \frac{x t^{3}}{6} x
\end{aligned}
$$

where the lower and the upper bounds exact solution of Equation (31) in fuzzy single parametric can be obtained by substituting $b=0$ and $b=1$ respectively in Equation (34). Next, from the analysis of HPM in Sections 4 and 6, we yield the following:

$$
\begin{aligned}
& p^{0}:\left\{b \bar{u}_{0}(x, t, r)-\underline{u}_{0}(x, t, r)+u_{0}(x, t, r)\right. \\
& =b[(2-2 r) x]+(r-1) x+b\left[(2-2 r) x^{2} t\right]+r x^{2} t \\
& p^{1}:\left\{\begin{array}{c}
b\left(\bar{u}_{1}(x, t, r)-\underline{u}_{1}(x, t, r)\right)+\underline{u}_{1}(x, t, r)=\int_{0}^{T} \int_{0}^{T}\left[\begin{array}{c}
-b\left(\frac{\partial^{2}}{\partial x^{2}} \bar{u}_{0}(x, t, r)-\frac{\partial^{2}}{\partial x^{2}} \underline{u}_{0}(x, t, r)\right)- \\
\frac{\partial^{2}}{\partial x^{2}} \underline{u}_{0}(x, t, r)+b((2-2 r) x t)+(r+1) x t
\end{array}\right] d t d t \\
\tilde{u}_{1}(x, 0, r, b)=0, \frac{\partial}{\partial t} \tilde{u}_{1}(x, 0, r, b)=0
\end{array}\right.
\end{aligned}
$$

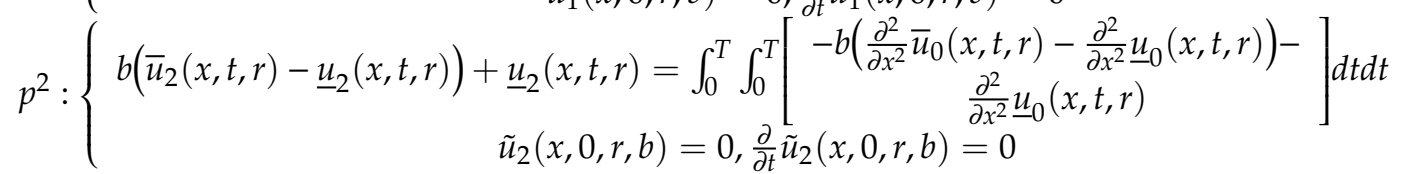

$$
\begin{aligned}
& p^{k+1}:\left\{\begin{array}{c}
b\left(\bar{u}_{k+1}(x, t, r)-\underline{u}_{k+1}(x, t, r)\right)+\underline{u}_{k+1}(x, t, r)=\int_{0}^{T} \int_{0}^{T}\left[\begin{array}{c}
-b\left(\frac{\partial^{2}}{\partial x^{2}} \bar{u}_{k}(x, t, r)-\frac{\partial^{2}}{\partial x^{2}} \underline{u}_{k}(x, t, r)\right)- \\
\frac{\partial^{2}}{\partial x^{2}} \underline{u}_{0}(x, t, r)
\end{array}\right] d t d t \\
\tilde{u}_{k+1}(x, 0, r, b)=0, \frac{\partial}{\partial t} \tilde{u}_{k+1}(x, 0, r, b)=0
\end{array}\right.
\end{aligned}
$$

In this example we define the fifth and tenth order double parametric fuzzy HPM approximate series solution of Equation (31) as follows:

$$
\tilde{u}(x, t, r, b)_{5,10}=\sum_{i=0}^{5,10} b\left(\bar{u}_{i}(x, t, r)-\underline{u}_{i}(x, t, r)\right)+\underline{u}_{i}(x, t, r) .
$$


By using Mathematica 12 software code, we obtain the corresponding fifth and tenth order series solution from Equation (35) as follows:

$$
\begin{aligned}
\tilde{u}(x, t, r, b)_{5}= & \left\{0.578125,(-1+r) x+\frac{1}{120}(-2 b(-1+r)+r) t^{5} x^{2}+\frac{(2 b(-1+r)-r) t^{7} x^{2}}{5040}\right. \\
& +\frac{(-2 b(-1+r)+r) t^{9} x^{2}}{362880}+\frac{(2 b(-1+r)-r) t^{11} x^{2}}{39916800}+b((1-r) x-(-1 \\
& +r) x) \frac{1}{6} t^{3} x\left(1+r+2 b(-1+r)(-1+x)-r x+t\left(r x^{2}+b\left((2-r) x^{2}\right.\right.\right. \\
& \left.-r x^{2}\right\}, \\
\tilde{u}(x, t, r, b)_{10}= & \left\{0.84375,(-1+r) x+\frac{1}{120}(-2 b(-1+r)+r) t^{5} x^{2}+\frac{(2 b(-1+r)-r) t^{7} x^{2}}{5040}\right. \\
& +\frac{(-2 b(-1+r)+r) t^{9} x^{2}}{362880}+\frac{(2 b(-1+r)-r) t^{11} x^{2}}{39916000} \\
& +\frac{(-2 b(-1+r)+r) t^{13} x^{2}}{6227020800}+\frac{(2 b(-1+r)-r) t^{15} x^{2}}{1307674368000} \\
& +\frac{(-2 b(-1+r)+r) t^{17} x^{2}}{355687428096000}+\frac{(2 b(-1+r)-r) t^{9} x^{2}}{121645100408832000} \\
& +\frac{(-2 b(-1+r)+r) t^{1} x^{2}}{51090942171709440000}+b((1-r) x-(-1+r) x)+\frac{1}{6} t^{3} x(1+r \\
& +2 b(-1+r)(-1+x)-r x)+t\left(r x^{2}+b\left(\left(2-r x^{2}-r x^{2}\right)\right)\right\} .
\end{aligned}
$$

From the above solutions the fifth and tenth order HPM double parametric solitons involves extra parameters $r$ and $b$ with two independent variables $t$ and $x$ but, even with these parameters it is found that the fifth order solution $\tilde{u}(x, t, r, b)_{5}$ is obtained within $0.578125 \mathrm{~s}$ while the tenth order solution $\tilde{u}(x, t, r, b)_{10}$ is obtained within $0.84375 \mathrm{~s}$ and that's enough of prove that the double parametric fuzzy number approximation approach based on HPM is a fast method with respect to CPU time consumption for solving fuzzy wave-like equation in the double parametric fuzzy number approach. From Section 8, the convergence of double parametric fuzzy HPM solution of Equation (24) can be shown by in case the values of $b=0$ and $r=0.2$ in Equation (35) to obtain the following:

$$
\begin{gathered}
\tilde{u}_{0}(x, t, 0.2,0)=-\frac{4 x}{5}+\frac{t x^{2}}{5} \\
\tilde{u}_{1}(x, t, 0.2,0)=-\frac{1}{30} t^{3}(-6+x) x \\
\tilde{u}_{2}(x, t, 0.2,0)=\frac{t^{5} x^{2}}{600} \\
\tilde{u}_{3}(x, t, 0.2,0)=-\frac{t^{7} x^{2}}{255200} \\
\tilde{u}_{4}(x, t, 0.2,0)=\frac{t^{9} x^{2}}{1814400}, \\
\tilde{u}_{5}(x, t, 0.2,0)=-\frac{t^{11} x^{2}}{19954000} \\
\tilde{u}_{6}(x, t, 0.2,0)=\frac{t^{1} x^{2}}{31135104000} \\
\cdots \\
\tilde{u}(x, t, 0.2,0)_{n}=\left(-\frac{4 x}{5}+\frac{t^{3} x}{5}+\frac{1}{5} x^{2} \sum_{n=0}^{\infty} \frac{(-1)^{n} t^{2 n+1}}{(2 n+1) !}\right) .
\end{gathered}
$$

From Equation (34), the exact solution of Equation (31) for $b=0$ and $r=0.2$ is given by $\tilde{U}(x, t, 0.2,0)=-\frac{4 x}{5}+\frac{t^{3} x}{5}+\frac{1}{5} x^{2} \sin (t)$. Then, from the convergence theorem in Section 8 , we have the following relation:

$$
\lim _{n \rightarrow \infty}\left\|\left(2 t+x^{2} \sum_{n=0}^{\infty} \frac{(-1)^{n} t^{2 n+1}}{(2 n+1) !}\right)-\left(2 t+2 \mathrm{e}^{-2 t} x^{2}\right)\right\|=0 \rightarrow \lim _{n \rightarrow \infty}\left(2 t+x^{2} \sum_{n=0}^{\infty} \frac{(-1)^{n} t^{2 n+1}}{(2 n+1) !}\right)=\left(2 t+2 \mathrm{e}^{-2 t} x^{2}\right),
$$

where $\lim _{n \rightarrow \infty}\left(\sum_{n=0}^{\infty} \frac{(-1)^{n} t^{2 n+1}}{(2 n+1) !}\right)=\sin (t)$. Thus, in the same manner, the double parametric fuzzy HPM converges to the exact solution of Equation (31) for each $r, b \in[0,1]$. In this example, the error analysis of double parametric HPM series solution of Equation (31) for all $r, b \in[0,1]$ is defined by the absolute errors between the exact solution (34) and the approximate solution (28) in the following form:

$$
\tilde{E}(x, t, r, b)_{5,10}=\left|\tilde{U}(x, t, r, b)-\tilde{u}(x, t, r, b)_{5,10}\right| .
$$


The numerical results in double parametric form obtained by fifth and tenth order HPM are compared with the exact solutions of Equation (31) for different values of $r, b \in[0,1]$ when $x=1$ and $t=\pi$ are showed in Tables 7-12. Also the double parametric form exact and approximate solutions of Equation (31) for different values $r, b \in[0,1]$ when $x=1$ and $t=\pi$ are also shown graphically in Figure 7.

Table 7. Solution and accuracy of fifth and tenth order double parametric fuzzy HPM solutions for Equation (32) at $t=\pi, x=1$ and $b=0$ for all $r \in[0,1]$.

\begin{tabular}{cccccc}
\hline $\boldsymbol{r}$ & $\boldsymbol{u}(1, \boldsymbol{\pi}, 0, \boldsymbol{r})_{5}$ & $\boldsymbol{u}(1, \boldsymbol{\pi}, 0, \boldsymbol{r})_{10}$ & $\boldsymbol{U}(1, \boldsymbol{\pi}, 0, \boldsymbol{r})$ & $\boldsymbol{E}(1, \boldsymbol{\pi}, 0, \boldsymbol{r})_{5}$ & $\boldsymbol{E}(1, \boldsymbol{\pi}, 0, \boldsymbol{r})_{10}$ \\
\hline 0 & 4.167712780049969 & 4.1677127800499690 & 4.1677127800499690 & 0 & 0 \\
0.2 & 5.401166304012321 & 5.4012553360620320 & 5.4012553360599630 & 0.00008903204 & $2.0694 \times 10^{-12}$ \\
0.4 & 6.634619827974673 & 6.6347978920740950 & 6.6347978920699560 & 0.00017806409 & $4.1389 \times 10^{-12}$ \\
0.6 & 7.868073351937026 & 7.8683404480861600 & 7.8683404480799500 & 0.00026709614 & $6.2101 \times 10^{-12}$ \\
0.8 & 9.101526875899378 & 9.1018830040982230 & 9.1018830040899450 & 0.00035612819 & $8.2778 \times 10^{-12}$ \\
1 & 0.334980399861730 & 10.335425560110288 & 10.335425560099939 & 0.00044516023 & $1.0349 \times 10^{-11}$ \\
\hline
\end{tabular}

Table 8. Solution and accuracy of fifth and tenth order double parametric fuzzy HPM solutions for Equation (32) at $t=\pi, x=1$ and $b=0.2$ for all $r \in[0,1]$.

\begin{tabular}{cccccc}
\hline $\boldsymbol{r}$ & $\boldsymbol{u}(1, \pi, 0.2, \boldsymbol{r})_{5}$ & $\boldsymbol{u}(1, \boldsymbol{\pi}, 0.2, \boldsymbol{r})_{10}$ & $\boldsymbol{U}(1, \boldsymbol{\pi}, 0.2, \boldsymbol{r})$ & $\boldsymbol{E}(1, \boldsymbol{\pi}, 0.2, \boldsymbol{r})_{5}$ & $\begin{array}{c}\boldsymbol{E}(1, \boldsymbol{\pi}, 0.2, \boldsymbol{r})_{10} \\
-\end{array}$ \\
\hline 0 & -6.6346198279746735 & 6.6347978920740970 & 6.634797892069958 & 0.000178064095 & $4.1389 \times 10^{-12}$ \\
0.2 & 7.3746919423520840 & 7.3749234256813345 & 7.374923425675952 & 0.000231483323 & $5.3823 \times 10^{-12}$ \\
0.4 & 8.1147640567294950 & 8.1150489592885720 & 8.115048959281950 & 0.000284902552 & $6.6222 \times 10^{-12}$ \\
0.6 & 8.8548361711069070 & 8.8551744928958110 & 8.855174492887945 & 0.000338321781 & $7.8657 \times 10^{-12}$ \\
0.8 & 9.5949082854843190 & 9.5953000265030490 & 9.595300026493943 & 0.000391741009 & $9.1056 \times 10^{-12}$ \\
1 & 10.334980399861730 & 10.335425560110288 & 10.335425560099939 & 0.000445160238 & $1.0349 \times 10^{-11}$ \\
\hline
\end{tabular}

Table 9. Solution and accuracy of fifth and tenth order double parametric fuzzy HPM solutions for Equation (32) at $t=\pi, x=1$ and $b=0.4$ for all $r \in[0,1]$.

\begin{tabular}{cccccc}
\hline $\boldsymbol{r}$ & $\boldsymbol{u}(1, \pi, 0.4, \boldsymbol{r})_{5}$ & $\boldsymbol{u}(1, \boldsymbol{\pi}, 0.4, \boldsymbol{r})_{10}$ & $\boldsymbol{U}(1, \boldsymbol{\pi}, 0.4, \boldsymbol{r})$ & $\boldsymbol{E}(1, \boldsymbol{\pi}, 0.4, \boldsymbol{r})_{5}$ & $\boldsymbol{E}(1, \boldsymbol{\pi}, 0.4, \boldsymbol{r})_{10}$ \\
\hline 0 & 9.101526875899378 & 9.101883004098223 & 9.101883004089945 & 0.000356128190 & $8.2778 \times 10^{-12}$ \\
0.2 & 9.348217580691848 & 9.348591515300637 & 9.348591515291943 & 0.000373934600 & $8.6934 \times 10^{-12}$ \\
0.4 & 9.594908285484319 & 9.595300026503047 & 9.595300026493943 & 0.000391741009 & $9.1038 \times 10^{-12}$ \\
0.6 & 9.841598990276790 & 9.842008537705460 & 9.842008537695941 & 0.000409547419 & $9.5194 \times 10^{-12}$ \\
0.8 & 10.08828969506925 & 10.088717048907874 & 10.088717048897940 & 0.000427353828 & $9.9333 \times 10^{-12}$ \\
1 & 10.33498039986173 & 10.335425560110288 & 10.335425560099939 & 0.000445160238 & $1.0349 \times 10^{-11}$ \\
\hline
\end{tabular}

Table 10. Solution and accuracy of fifth and tenth order double parametric fuzzy HPM solutions for Equation (32) at $t=\pi, x=1$ and $b=0.6$ for all $r \in[0,1]$.

\begin{tabular}{cccccc}
\hline $\boldsymbol{r}$ & $\overline{\boldsymbol{u}}(1, \boldsymbol{\pi}, 0.6, \boldsymbol{r})_{5}$ & $\overline{\boldsymbol{u}}(1, \boldsymbol{\pi}, 0.6, \boldsymbol{r})_{10}$ & $\overline{\boldsymbol{U}}(1, \boldsymbol{\pi}, 0.6, \boldsymbol{r})$ & $\overline{\boldsymbol{E}}(1, \boldsymbol{\pi}, 0.6, \boldsymbol{r})_{5}$ & $\overline{\boldsymbol{E}}(1, \boldsymbol{\pi}, 0.6, \boldsymbol{r})_{10}$ \\
\hline 0 & 11.5684339238240830 & 11.56896811612235 & 11.568968116109932 & 0.000534192285 & $1.2418 \times 10^{-11}$ \\
0.2 & 11.32174321903161 & 11.322259604919937 & 11.322259604907932 & 0.000516385876 & $1.2004 \times 10^{-11}$ \\
0.4 & 11.075052514239141 & 11.075551093717525 & 11.075551093705934 & 0.000498579466 & $1.1590 \times 10^{-11}$ \\
0.6 & 10.828361809446670 & 10.828842582515113 & 10.828842582503935 & 0.000480773057 & $1.1178 \times 10^{-11}$ \\
0.8 & 10.581671104654202 & 10.582134071312698 & 10.582134071301937 & 0.000462966647 & $1.0761 \times 10^{-11}$ \\
1 & 10.334980399861730 & 10.335425560110288 & 10.335425560099939 & 0.000445160238 & $1.0349 \times 10^{-11}$ \\
\hline
\end{tabular}

Table 11. Solution and accuracy of fifth and tenth order double parametric fuzzy HPM solutions for Equation (32) at $t=\pi, x=1$ and $b=0.8$ for all $r \in[0,1]$.

\begin{tabular}{cccccc}
\hline $\boldsymbol{r}$ & $\overline{\boldsymbol{u}}(1, \boldsymbol{\pi}, 0.8, \boldsymbol{r})_{5}$ & $\overline{\boldsymbol{u}}(1, \boldsymbol{\pi}, 0.8, \boldsymbol{r})_{10}$ & $\overline{\boldsymbol{U}}(1, \boldsymbol{\pi}, 0.8, \boldsymbol{r})$ & $\overline{\boldsymbol{E}}(1, \boldsymbol{\pi}, 0.8, \boldsymbol{r})_{5}$ & $\overline{\boldsymbol{E}}(1, \boldsymbol{\pi}, 0.8, \boldsymbol{r})_{10}$ \\
\hline 0 & 14.035340971748784 & 14.036053228146478 & 14.036053228129921 & 0.000712256381 & $1.6557 \times 10^{-11}$ \\
0.2 & 13.295268857371376 & 13.295927694539241 & 13.295927694523924 & 0.000658837152 & $1.5317 \times 10^{-11}$ \\
0.4 & 12.555196742993962 & 12.555802160932 & 12.555802160917928 & 0.000605417923 & $1.4072 \times 10^{-11}$ \\
0.6 & 11.815124628616552 & 11.815676627324763 & 11.815676627311930 & 0.000551998695 & $1.2832 \times 10^{-11}$ \\
0.8 & 11.075052514239141 & 11.075551093717525 & 11.075551093705934 & 0.000498579466 & $1.1590 \times 10^{-11}$ \\
1 & 10.33498039986173 & 10.335425560110288 & 10.335425560099939 & 0.000445160238 & $1.0349 \times 10^{-11}$ \\
\hline
\end{tabular}


Table 12. Solution and accuracy of fifth and tenth order double parametric fuzzy HPM solutions for Equation (32) at $t=\pi, x=1$ and $b=1$ for all $r \in[0,1]$.

\begin{tabular}{cccccc}
\hline $\boldsymbol{r}$ & $\overline{\boldsymbol{u}}(1, \pi, 1, \boldsymbol{r})_{5}$ & $\overline{\boldsymbol{u}}(1, \pi, 1, \boldsymbol{r})_{10}$ & $\overline{\boldsymbol{U}}(1, \pi, 1, \boldsymbol{r})$ & $\overline{\boldsymbol{E}}(1, \pi, 1, \boldsymbol{r})_{5}$ & $\overline{\boldsymbol{E}}(1, \pi, 1, \boldsymbol{r})_{10}$ \\
\hline 0 & 16.502248019673488 & 16.503138340170604 & 16.50313834014991 & 0.000890320476 & $2.0694 \times 10^{-11}$ \\
0.2 & 15.268794495711138 & 15.269595784158541 & 15.269595784139913 & 0.000801288428 & $1.8628 \times 10^{-11}$ \\
0.4 & 14.035340971748782 & 14.036053228146478 & 14.036053228129921 & 0.000712256381 & $1.6557 \times 10^{-11}$ \\
0.6 & 12.801887447786434 & 12.802510672134412 & 12.802510672119926 & 0.000623224333 & $1.4486 \times 10^{-11}$ \\
0.8 & 11.568433923824081 & 11.568968116122350 & 11.568968116109932 & 0.000534192285 & $1.2418 \times 10^{-11}$ \\
1 & 10.33498039986173 & 10.335425560110288 & 10.335425560099939 & 0.000445160238 & $1.0349 \times 10^{-11}$ \\
\hline
\end{tabular}

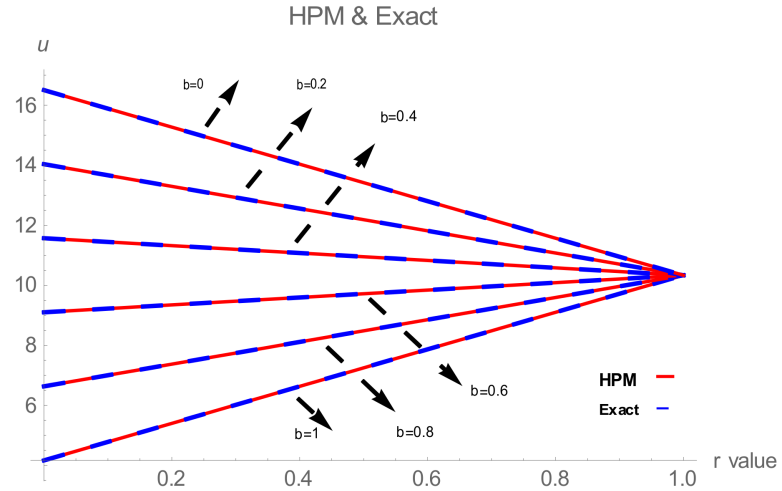

Figure 7. Comparison of the exact and tenth-order double parametric fuzzy HPM solutions of Equation (31) at $t=\pi, x=1$ for different values of $r, b \in[0,1]$.

Here in Tables 7-12 and Figure 7, all the solutions for $b=0,0.2,0.4$ gives the lower fuzzy solutions of Equation (32) while the solutions for $b=0.6,0.8,1$ give the upper fuzzy solution of Equation (31) for all $r \in[0,1]$ satisfy the properties of triangular fuzzy numbers. Clearly that the tenth order double parametric fuzzy HPM series solution converges with higher accuracy to the exact solution of Equation (31).
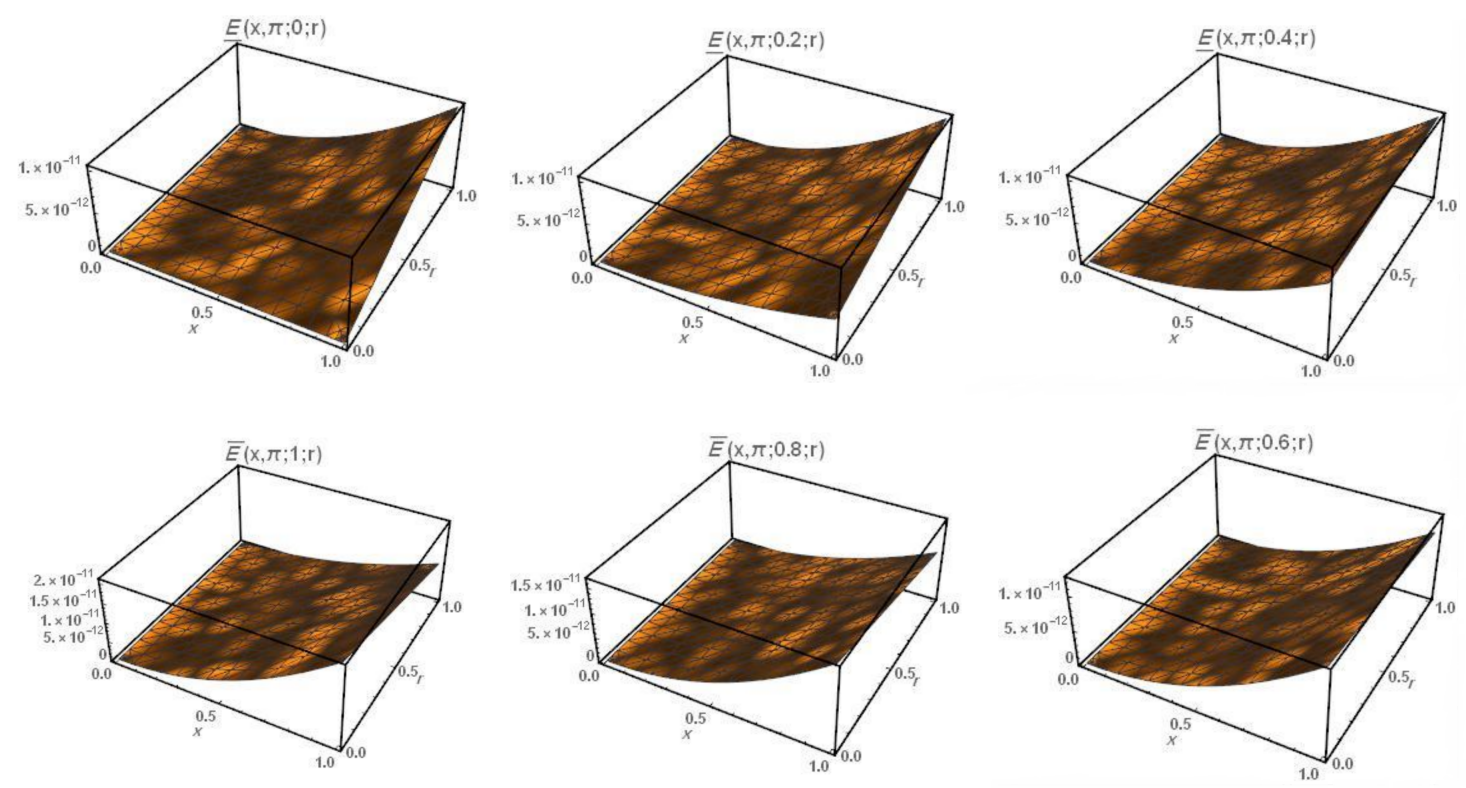

Figure 8. Accuracy of the tenth-order double parametric fuzzy HPM solution at $t=\pi, b=$ $0,0.2,0.4,0.6,0.8,1$ for all $x \in[0,1]$ and $r \in[0,1]$.

Figure 8 is used to show the accuracy of fifth order double parametric fuzzy HPM upper and lower solutions of Equation (31) in three-dimensional shape for $t=\pi b$ over the interval $x \in[0,1]$ 
and different values of $b=0,0.2,0.4,0.6,0.8,1$ for all $r \in[0,1]$. Moreover the fifth order double parametric HPM approximate solutions of Equation (31) all $x \in[0,1]$ and $r \in[0,1]$ different values of $b=0,0.2,0.4,0.6,0.8,1$ when $t=\pi$ are presented graphically in Figures 8-11.

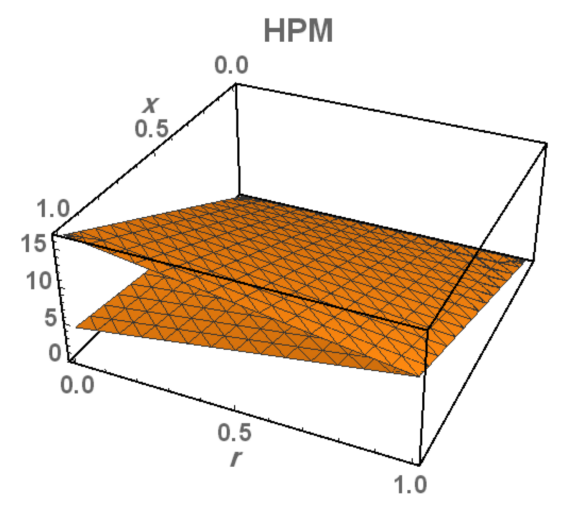

Figure 9. Solution of the tenth-order double parametric fuzzy HPM at $t=\pi, b=0,1$ for all $x \in[0,1]$ and $r \in[0,1]$.

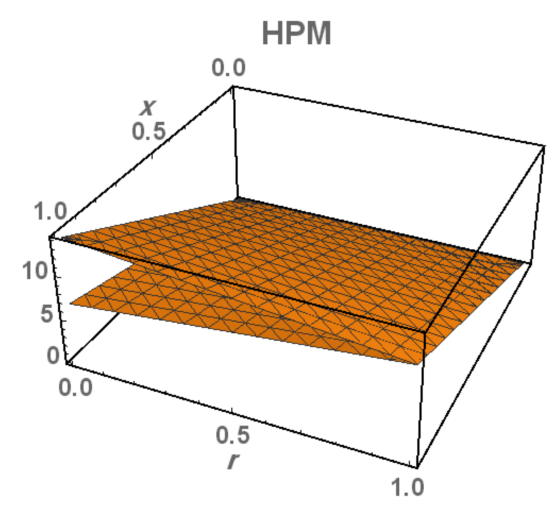

Figure 10. Solution of the tenth-order double parametric fuzzy HPM at $t=\pi, b=0.2,0.8$ for all $x \in[0,1]$ and $r \in[0,1]$.

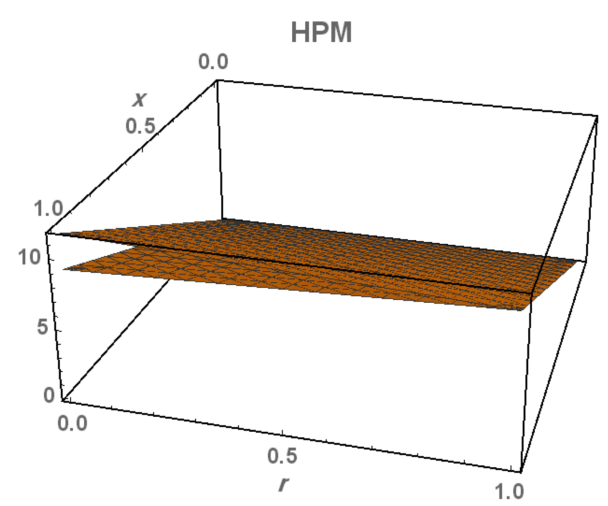

Figure 11. Solution of the tenth-order double parametric fuzzy HPM at $t=\pi, b=0.4,0.6$ for all $x \in[0,1]$ and $r \in[0,1]$.

Figures 8-11 show that the tenth order double parametric fuzzy HPM solutions of Equation (24) for all $x \in[0,1]$ and $r \in[0,1]$ of different values $b=0,0.2,0.4,0.6,0.8,1$ when $t=\pi$ satisfy the properties of triangular fuzzy numbers. 


\section{Conclusions}

We successfully developed an approximate scheme for solving one-dimensional heat-like and wave-like equations in a fuzzy environment based on the HPM. In the formulation of the developed double parametric fuzzy HPM, the equations are first converted into interval-based fuzzy differential equations. Subsequently, the resulting differential equations are transformed by applying the double parametric approach using the embedding parameter where this parameter $b$, which deforms from 0 to 1 , reduces the computational and analysis work to obtain the solutions. The convergence of the solutions was proven, and the findings from numerical examples signify the accuracy and efficiency of the double parametric fuzzy HPM developed in this study.

Author Contributions: Conceptualization, A.F.J.; methodology, A.F.J., S.A.J.A. and S.G.A.A.; software, A.F.J. and S.A.J.A.; writing-review \& editing, A.A. and N.H.M. All authors had equal contributions in this article. All authors have read and agreed to the published version of the manuscript.

Funding: This research has been funded by the Fundamental Research Grants Scheme (FRGS) given by the Ministry of Higher Education of Malaysia S/O number 14188.

Acknowledgments: The authors are extremely grateful to the Universiti Utara Malaysia and the Ministry of Higher Education of Malaysia for the Fundamental Research Grants Scheme (FRGS) S/O number 14188 for the support of this research and also to Associate Azizan Bin Saaban for his guidance and advice.

Conflicts of Interest: It is declared that the authors have no competing interests.

Availability of Data and Materials: There is no additional data required for the finding of results of this paper.

\section{References}

1. Atanassov, K.M. Intuitionistic Fuzzy Sets: Theory and Applications, Studies in Fuzziness and Soft Computing; Springer: Berlin/Heidelberg, Germany, 1999; Volume 35.

2. Zimmermann, H.-J. Fuzzy Set Theory-And Its Applications; Springer Science: New York, NY, USA, 2001.

3. Bede, B. Note on Numerical solutions of fuzzy differential equations by predictor corrector method. Inf. Sci. 2008, 178, 1917-1922. [CrossRef]

4. Bertone, A.M.; Jafelice, R.M.; de Barros, L.C.; Bassanezi, R.C. On fuzzy solutions for partial differential equations. Fuzzy Set Syst. 2013, 219, 68-80. [CrossRef]

5. Buckley, J.J.; Feuring, T. Introduction to fuzzy partial differential equations. Fuzzy Set Syst. 1999, 105, $241-248$. [CrossRef]

6. Jafari, R.; Yu, W. Fuzzy Control for Uncertainty Nonlinear Systems with Dual Fuzzy Equations. J. Intell. Fuzzy Syst. 2015, 29, 1229-1240. [CrossRef]

7. Mikaeilvand, N.; Khakrangin, S. Solving fuzzy partial differential equations by fuzzy two-dimensional differential transform method. Neural Comput. Appl. 2013, 21, 307-312. [CrossRef]

8. Mohammad, M. Fuzzy-Stochastic Partial Differential Equations. SIAM-ASA J. Uncertain. Quantif. 2019, 7, 1076-1104.

9. Raheleh, J.; Sina, R. Solution of Fuzzy Differential Equations Using Fuzzy Sumudu Transforms. Math. Comput. Appl. 2018, 32,1-15.

10. Lalla, S.C.; Atimad, H.; Said, M. Exact fuzzy solution of the fuzzy heat-like equations. arXiv 2013, arXiv:1305.2633.

11. Melliani, S.; Harir, A.; Chadli, L.S. Solutions of fuzzy heat-like equations by variational iteration method. Ann. Fuzzy Math. Inform. 2015, 10, 29-44.

12. Rouhparvar, H.; Abbasbandy, S.; Allahviranloo, T. The variational iteration method for exact solutions of fuzzy heat-like equations with variable coefficients. J. Am. Sci. 2011, 7, 338-345.

13. Narayanamoorthy, S.; Murugan, K. Solutions of fuzzy heat-like equations by variational iteration method. Ann. Fuzzy Math. Inform. 2014, 7, 133-142.

14. Mawia, O.; Zengtai, G.; Altyeb, M.M. Comparison of fuzzy Adomian decomposition method with fuzzy VIM for solving fuzzy heat-like and wave-like equations with variable coefficients. Adv. Differ. Equ. 2020, $2020,327$.

15. Chadli, L.S.; Harir, A.; Melliani, S. Solutions of fuzzy wave-like equations by variational iteration method. Int. Ann. Fuzzy Math. Inform. 2014, 8, 527-547. 
16. Hashemi, M.S.; Malekinagad, J. Series solution of fuzzy wave-like equations with variable coefficients. J. Intell. Fuzzy Syst. 2013, 25, 415-428. [CrossRef]

17. Sarmad, A.A.; Ali, F.J.; Azizan, S. A Single Convergent Control Parameter Optimal Homotopy Asymptotic Method Approximate-Analytical Solution of Fuzzy Heat Equation. ASM Sci. J. 2019, 12, 42-47.

18. Pirzada, U.M.; Vakaskar, D.C. Solution of fuzzy heat equations using Adomian Decomposition method. Adv. Appl. Math. Mech. 2015, 3, 87-91.

19. Allahviranloo, T.; Abbasbandya, S.; Rouhparvarc, H. The exact solutions of fuzzy wave-like equations with variable coefficients by a variational iteration method. Appl. Soft Comput. 2011, 11, 2186-2192. [CrossRef]

20. Macias-Diaz, J.E.; Tomasiello, S. A differential quadrature-based approach à la Picard for systems of partial differential equations associated to fuzzy differential equations. J. Comput. Appl. Math. 2016, 299, 15-23. [CrossRef]

21. Biazar, J.; Ghazvini, H. Convergence of the Homotopy Perturbation Method for Partial Differential equations. Nonlinear Anal. Real World Appl. 2009, 10, 2633-2640. [CrossRef]

22. Chun, C.; Jafari, H.; Kim, Y.-I. Numerical method for the wave and non-linear diffusion equations with the homotopy perturbation method. Comput. Math. Appl. 2009, 57, 1226-1231. [CrossRef]

23. Gupta, A.K.; Saha, R.S. Comparison between homotopy perturbation method and optimal homotopy asymptotic method for the solution of Boussinesq-Burgers equations. Comput. Fluids 2014, 103, 34-41. [CrossRef]

24. He, J.-H. A coupling method of a homotopy technique and a perturbation technique for non-linear problems. Int. J. Nonlinear Mech. 2000, 35, 7-43. [CrossRef]

25. He, J.-H. Homotopy perturbation technique. Comput. Methods Appl. Mech. Eng. 1999, 178, $257-262$. [CrossRef]

26. Tapaswini, S.; Chakraverty, S. Numerical solution of uncertain beam equations using double parametric form of fuzzy numbers. Appl. Comput. Intell. Soft Comput. 2013, 2013, 100-111. [CrossRef]

27. Jameel, A.F.; Amen, S.G.; Saaban, A.; Man, N.H.; Alipiah, F.M. Homotopy Perturbation Method for Solving Linear Fuzzy Delay Differential Equations Using Double Parametric Approach. Math. Stat. 2020, 8, 551-558. [CrossRef]

28. Chen, Y.Y.; Chang, Y.T.; Chen, B.S. Fuzzy Solutions to Partial Differential Equations: Adaptive Approach. IEEE Trans. Fuzzy Syst. 2009, 17, 116-127. [CrossRef]

29. Bodjanova, S. Median Alpha-Levels of a Fuzzy Number. Fuzzy Set Syst. 2006, 157, 879-891. [CrossRef]

30. Diptiranjan., B.; Chakraverty, S. New approach to solve fully fuzzy system of linear equations using single and double parametric form of fuzzy numbers. Sadhana 2015, 40, 35-49.

31. Fard, O.S. An Iterative Scheme for the Solution of Generalized System of Linear Fuzzy Differential Equations. World Appl. Sci. J. 2009, 7, 1597-1604.

32. Guo, X.; Shang, D.; Lu, X. Fuzzy Approximate Solutions of Second-Order Fuzzy Linear Boundary Value Problems. Bound. Value Probl. 2013, 2013, 212. [CrossRef]

33. Xixiang, Z.; Weimin, M.; Chen, L. New Similarity of Triangular Fuzzy Number and Its Application. Sci. World J. 2014, 2014, 215047.

34. Dubois, D.; Prade, H. Towards fuzzy differential calculus, Part 3: Differentiation. Fuzzy Set Syst. 1982, 8, 225-233. [CrossRef]

35. Turkyilmazoglu, M. Convergence of the homotopy perturbation method. Int. J. Nonlinear Sci. Numer. Simul. 2011, 12, 9-14. [CrossRef]

Publisher's Note: MDPI stays neutral with regard to jurisdictional claims in published maps and institutional affiliations.

(C) 2020 by the authors. Licensee MDPI, Basel, Switzerland. This article is an open access article distributed under the terms and conditions of the Creative Commons Attribution (CC BY) license (http://creativecommons.org/licenses/by/4.0/). 